\title{
The Evolution of First Person Vision Methods: A Survey
}

\author{
A. Betancourt ${ }^{1,2}$, P. Morerio ${ }^{1}$, C.S. Regazzoni ${ }^{1}$, and M. Rauterberg ${ }^{2}$ \\ ${ }^{1}$ Department of Naval, Electric, Electronic and Telecommunications Engineering DITEN - University of Genova, Italy \\ ${ }^{2}$ Designed Intelligence Group, Department of Industrial Design, Eindhoven University of Technology, Eindhoven, Netherlands
}

\begin{abstract}
The emergence of new wearable technologies such as action cameras and smart-glasses has increased the interest of computer vision scientists in the First Person perspective. Nowadays, this field is attracting attention and investments of companies aiming to develop commercial devices with First Person Vision recording capabilities. Due to this interest, an increasing demand of methods to process these videos, possibly in real-time, is expected. Current approaches present a particular combinations of different image features and quantitative methods to accomplish specific objectives like object detection, activity recognition, user machine interaction and so on. This paper summarizes the evolution of the state of the art in First Person Vision video analysis between 1997 and 2014, highlighting, among others, most commonly used features, methods, challenges and opportunities within the field.
\end{abstract}

Index Terms-First Person Vision, Egocentric Vision, Wearable Devices, Smart-Glasses, Computer Vision, Video Analytics, Humanmachine Interaction.

\section{INTRODUCTION}

Portable head-mounted cameras, able to record dynamic high quality first-person videos, have become a common item among sportsmen over the last five years. These devices represent the first commercial attempts to record experiences from a first-person perspective. This technological trend is a follow-up of the academic results obtained in the late 1990s, combined with the growing interest of the people to record their daily activities. Up to now, no consensus has yet been reached in literature with respect to naming this video perspective. First Person Vision (FPV) is arguably the most commonly used, but other names, like Egocentric Vision or Ego-vision has also recently grown in popularity. The idea of recording and analyzing videos from this perspective is not new in fact, several such devices have been developed for research purposes over the last 15 years $[1,2,3,4,5]$. Figure 1 shows the growth in the number of articles related to FPV video analysis between 1997 and 2014. Quite remarkable is the seminal work carried out by the Media lab (MIT) in the late 1990s and early 2000s $[6,7,8,9,10,11]$, and the multiple devices proposed by Steve

a.betancourt@tue.nl

pietro.morerio@ginevra.dibe.unige.it

carlo@dibe.unige.it

g.w.m.rauterberg@tue.nl

This work was supported in part by the Erasmus Mundus joint Doctorate in Interactive and Cognitive Environments, which is funded by the EACEA, Agency of the European Commission under EMJD ICE.

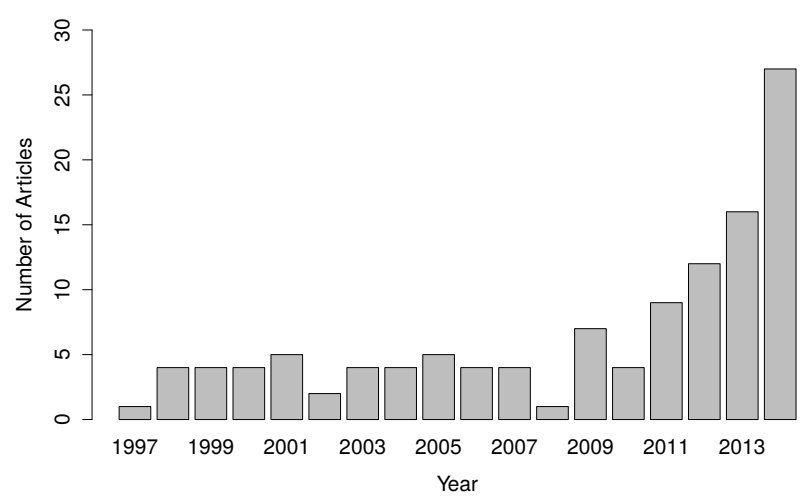

Fig. 1. Number of articles per year directly related to FPV video analysis. This plot contains the articles published until 2014, to the best of our knowledge

Mann who, back in 1997 [12], described the field with these words :

"Let's imagine a new approach to computing in which the apparatus is always ready for use because it is worn like clothing. The computer screen, which also serves as a viewfinder, is visible at all times and performs multi-modal computing (text and images)".

Recently, in the awakening of this technological trend, several companies have been showing interest in this kind of devices (mainly smart-glasses), and multiple patents have been presented. Figure 1 shows the devices patented in 2012 by Google and Microsoft. Together with its patent, Google also announced Project Glass, as a strategy to test its device among a exploratory group of people. The project was introduced by showing short previews of the Glasses' FPV recording capabilities, and its ability to show relevant information to the user through the head-up display.

Remarkably, the impact of the Glass Project (wich the most significant attempt to commercialize wearable technology up to date) is to be ascribed not only to its hardware, but also to the appeal of its underlying operating system. The latter continues to bring a large group of skilled developers, thus in turn making a significant boost in the number of prospective applications for smart-glasses, a phenomenon that has happened with smartphones several years ago. On one hand, the range of application fields that could benefit from smart-glasses 


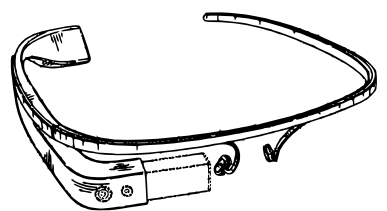

(a) Google glasses (U.S. Patent D659,741 - May 15, 2012).

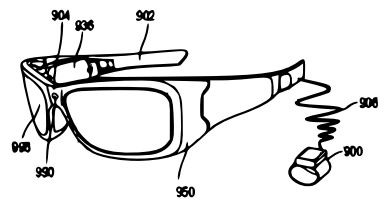

(b) Microsoft augmented reality glasses (U.S. Patent Application 20120293548 - Nov 22, 2012).

Fig. 2. Examples of the commercial smart patents. (a) Google patent of the smart-glasses; (b) Microsoft patent of an augmented reality wearable device.

is wide and applications are expected in areas like military strategy, enterprise applications, tourist services [13], massive surveillance [14], medicine [15], driving assistance [16], among others. On the other hand, what was until now considered as a consolidated research field, needs to be re-evaluated and restated under the light of this technological trend: wearable technology and the first person perspective rise important issues, such as privacy and battery life, in addition to new algorithmic challenges [17].

This paper summarizes the state of the art in FPV video analysis and its temporal evolution between 1997 and 2014, analyzing the challenges and opportunities of this video perspective. It reviews the main characteristics of previous studies using tables of references, and the main events and relevant works using timelines. As an example, Figure 3 presents some of the most important papers and commercial announcements in the general evolution of FPV. We direct interested readers to the must read papers presented in this timeline. In the following sections, more detailed timelines are presented according to the objective addressed in the summarized papers. The categories and conceptual groups presented in this survey reflects our schematic perception of the field coming from a detailed study of the existent literature. We are confident that the proposed categories are wide enough to conceptualize existent methods, however due to the growing speed of the field they could require future updates. As will be shown in the coming sections, the strategies used during the last 20 years are very heterogeneous. Therefore, rather than provide a comparative structure between existing methods and features, the objective of this paper is to highlight common points of interest and relevant future lines of research. The bibliography presented in this paper is mainly in FPV. However, some particular works in classic video analysis are also mentioned to support the analysis. The latter are cited using italic font as a visual cue.

To the best of our knowledge, the only paper summarizing the general ideas of the FPV is [18], which presents a wearable device and several possible applications. Other related reviews include the following: [19] reviews the activity recognition methods with multiple sensors; [20] analyzes the use of wearable cameras for medical applications; [3] presents some challenges of an active wearable device.

In the remainder of this paper, we summarize existent methods in FPV, according to a hierarchical structure we propose, highlighting the more relevant works and the temporal evolution of the field. Section 2 introduces general characteristics of FPV and the hierarchical structure, which is later used to summarize the current methods according to their final objective, the sub- tasks performed and the features used. In section 3 we briefly present the publicly-available FPV datasets. Finally, section 4 discusses some future challenges and research opportunities in this field.

\section{First Person Vision (FPV) VIDEO ANALYSIS}

During the late 1990s and early 2000s, the advances in FPV analysis were mainly performed using highly elaborated devices, typically proprietarily developed by different research groups. The list of devices proposed is wide, where each device was usually presented in conjunction with their potential applications and a large array of sensors which only envy from modern devices in their design, size and commercial capabilities. The column "Hardware" in Table 2 summarizes these devices. The remaining columns of this table are explained in section 2.1. Nowadays, current devices could be considered as the embodiment of the futuristic perspective of the already mentioned pioneering studies. Table 1 shows the currently available commercial projects and their embedded sensors. Such devices are grouped in three categories:

- Smart-glasses: Smart-glasses have multiple sensors, processing capabilities and a head-up display, making them ideal to develop real time methods and to improve the interaction between the user and its device. Besides, smartglasses are nowadays seen as the starting point of an augmented reality system. However, they cannot be considered a mature product until major challenges, such as battery life, price and target market, are solved. The future of these devices is promising, but it is still not clear if they will be adopted by the users on a daily basis like smartphones, or whether they will become specialized task-oriented devices like industrial glasses, smarthelmets, sport devices, etc.

- Action cameras: commonly used by sportsmen and lifeloggers. However, the research community has been using them as a tool to develop methods and algorithms while anticipating the commercial availability of the smartglasses during the coming years. Action cameras are becoming cheaper, and are starting to exhibit (albeit still somewhat limited) processing capabilities.

- Eye trackers: have been successfully applied to analyze consumer behaviors in commercial environments. Prototypes are available mainly for research purposes, where multiple applications have been proposed in conjunction with FPV. Despite the potential of these devices, their popularity is highly affected by the price of their components and the obtrusiveness of the eye tracker sensors, which is commonly carried out using an eye pointing camera.

FPV video analysis gives some methodological and practical advantages, but also inherently brings a set of challenges that need to be addressed [18]. On one hand, FPV solves some problems of the classical video analysis and offers extra information:

- Videos of the main part of the scene: Wearable devices allow the user to (even unknowingly) record the most relevant parts of the scene for the analysis, thus reducing the necessity for complex controlled multi-camera systems [23]. 
[12] Steve Mann presents some experiments with wearable devices.
[4] Microsoft Research releases the SenseCam.
[22] Brief summary of the devices proposed by Seteve Mann.

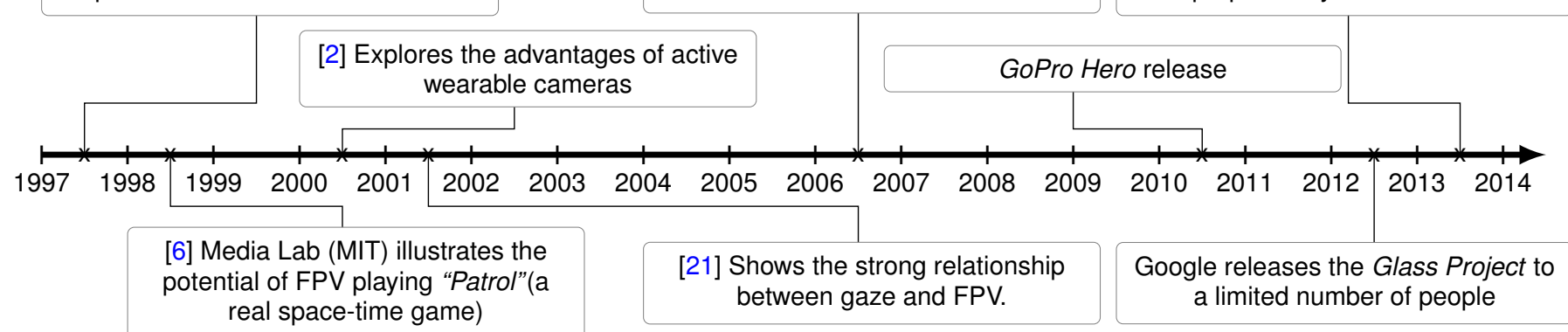

Fig. 3. Some of the more important works and commercial announcements in FPV.

TABLE 1

Commercial approaches to wearable devices with FPV video recording capabilities

\begin{tabular}{|c|c|c|c|c|c|c|c|c|c|c|c|c|c|}
\hline & 莺 & 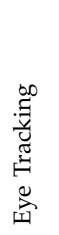 & 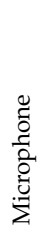 & $\hat{U}_{0}^{\infty}$ & 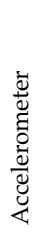 & 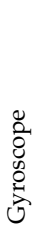 & 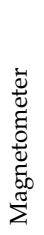 & $\underset{⿱ 乛}{\stackrel{Z}{Z}}$ & 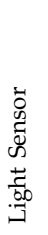 & 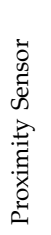 & 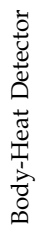 & 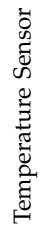 & 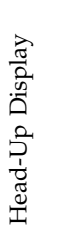 \\
\hline Google Glasses & $\checkmark$ & & $\checkmark$ & $\checkmark$ & $\checkmark$ & $\checkmark$ & $\checkmark$ & & $\checkmark$ & $\checkmark$ & & & $\checkmark$ \\
\hline Epson Moverio & $\checkmark$ & & $\checkmark$ & $\checkmark$ & $\checkmark$ & $\checkmark$ & $\checkmark$ & & & & & & $\checkmark$ \\
\hline Recon Jet & $\checkmark$ & & $\checkmark$ & $\checkmark$ & $\checkmark$ & $\checkmark$ & $\checkmark$ & & & & & $\checkmark$ & $\checkmark$ \\
\hline Vuzix M100 & $\checkmark$ & & $\checkmark$ & & $\checkmark$ & $\checkmark$ & $\checkmark$ & & $\checkmark$ & $\checkmark$ & & & $\checkmark$ \\
\hline GlassUp & $\checkmark$ & & $\checkmark$ & & $\checkmark$ & $\checkmark$ & $\checkmark$ & & $\checkmark$ & & & & $\checkmark$ \\
\hline Meta & $\checkmark$ & & $\checkmark$ & $\checkmark$ & $\checkmark$ & $\checkmark$ & & & & & & & $\checkmark$ \\
\hline Optinvent Ora-s & $\checkmark$ & & $\checkmark$ & $\checkmark$ & $\checkmark$ & $\checkmark$ & $\checkmark$ & & $\checkmark$ & & & & $\checkmark$ \\
\hline SenseCam & $\checkmark$ & & $\checkmark$ & & $\checkmark$ & & & $\checkmark$ & $\checkmark$ & & $\checkmark$ & $\checkmark$ & \\
\hline Lumus & $\checkmark$ & & $\checkmark$ & & $\checkmark$ & $\checkmark$ & $\checkmark$ & & & & & & $\checkmark$ \\
\hline Pivothead & $\checkmark$ & & $\checkmark$ & & & & & & & & & & \\
\hline GoPro & $\checkmark$ & & $\checkmark$ & & & & & & $\checkmark$ & & & & \\
\hline Looxcie camera & $\checkmark$ & & $\checkmark$ & & & & & & & & & & \\
\hline Epiphany Eyewear & $\checkmark$ & & $\checkmark$ & & & & & & & & & & \\
\hline SMI Eye tracking Glasses & $\checkmark$ & $\checkmark$ & $\checkmark$ & & & & & & & & & & \\
\hline Tobii & $\checkmark$ & $\checkmark$ & $\checkmark$ & & & & & & & & & & \\
\hline \multicolumn{14}{|c|}{$\begin{array}{l}{ }^{1} \text { Other projects such as Orcam, Nissan, Telepathy, Olympus MEG4.0, Oculon and Atheer have been officially } \\
\text { announced by their producers but no technical specifications have been already presented. } \\
2 \text { According to unofficial online sources, other companies like Apple, Samsung, Sony, Oakley could be } \\
\text { working on their own versions of similar devices, however no information has been officially announced } \\
\text { up to date. Microsoft recently announced the Hololens but not technical specifications have been } \\
\text { officially presented. } \\
2 \text { This data is created on January } 2015 \text {. } \\
{ }^{3} \text { In [18] one multi-sensor device is presented for research purposes. }\end{array}$} \\
\hline
\end{tabular}

- Variability of the datasets: Due to the increasing commercial interest of the technology companies, a large number of FPV videos is expected in the future, making it possible for the researchers to obtain large datasets that differ among themselves significantly, as discussed in section 3 .

- Illumination and scene configuration: Changes in the illumination and global scene characteristics could be used as an important feature to detect the scene in which the user is involved, e.g. detecting changes in the place where the activity is taking place, as in [24].

- Internal state inference: According to [25], eye and head movements are directly influenced by the person's emotional state. As already done with smartphones [26], this fact can be exploited to infer the user's emotional state, and provide services accordingly.

- Object positions: Because users tend to see the objects while interacting with them, it is possible to take advantage of the prior knowledge of the hands' and objects' positions, e.g. active objects tend to be closer to the center, whereas hands tend to appear in the bottom left and bottom right part of the frames [27, 28].

On the other hand, FPV itself also presents multiple challenges, which particularly affect the choice of the features to be extracted by low level processing modules (feature selection is discussed in details in section 2.3):

- Non static cameras: One of the main characteristics of FPV videos is that cameras are always in movement. This fact makes it difficult to differentiate between the background and the foreground [29]. Camera calibration is not possible and often scale, rotation and/or translation-invariant features are required in higher level modules. 
- Illumination conditions: The locations of the videos are highly variable and uncontrollable (e.g. visiting a touristic place during a sunny day, driving a car at night, brewing coffee in the kitchen). This makes it necessary to deploy robust methods for dealing with the variability in illumination. Here shape descriptors may be preferred to colorbased features [28].

- Real time requirements: One of the motivations for FPV video analysis is its potential of being used for real time activities. This implies the need for the real time processing capabilities [30].

- Video processing: Due to the embedded processing capabilities (for smart-glasses), it is important to define efficient computational strategies to optimize battery life, processing power and communication limits among the processing units. At this point, cloud computing could be seen as the most promising candidate tool to turn the FPV video analysis into an applicable framework for daily use. However, a real time cloud processing strategy requires further development in video compressing methods and communication protocols between the device and the cloud processing units.

The rest of this chapter summarizes FPV video analysis methods according to a hierarchical structure, as shown in Figure 4, starting from the raw video sequence (bottom) to the desired objectives (top). Section 2.1 summarizes the existent approaches according to 6 general objectives (Level 1). Section 2.2 divides these objectives in 15 weakly dependent subtasks (Level 2). Section briefly introduces the most commonly used image features, presenting their advantages and disadvantages, and relating them with objectives. Finally, section 2.4 summarizes the quantitative and computational tools used to process data, moving from one level to the other. In our literature review, we found that existing methods are commonly presented as combinations of the aforementioned levels. However, no standard structure is presented, making it difficult for other researchers to replicate existing methods or improve the state of the art. We propose this hierarchical structure as an attempt to cope with this issue.

\subsection{Objectives}

Table 2 summarizes a total of 117 articles. The articles are divided in six objectives according to the main goal addressed in each of them. The left side of the table contains the six objectives described in this section, and on the right side, extra groups related to hardware, software, related surveys and conceptual articles, are given. The category named "Particular Subtasks" is used for articles focused on one of the subtasks presented in section 2.2. The last column shows the positive trend in the number of articles per year, and is plotted in Figure 1 .

Note from the table that the most commonly explored objective is Object Recognition and Tracking. We identify it as the base of more advanced objectives such as Activity Recognition, Video Summarization and Retrieval and Environment Mapping. Another often studied objective is User-Machine Interaction because of its potential in Augmented Reality. Finally, a recent research line denoted as Interaction Detection allows the devices to infer situations in which the user is involved. Along with this section, we present some details of how existent methods have

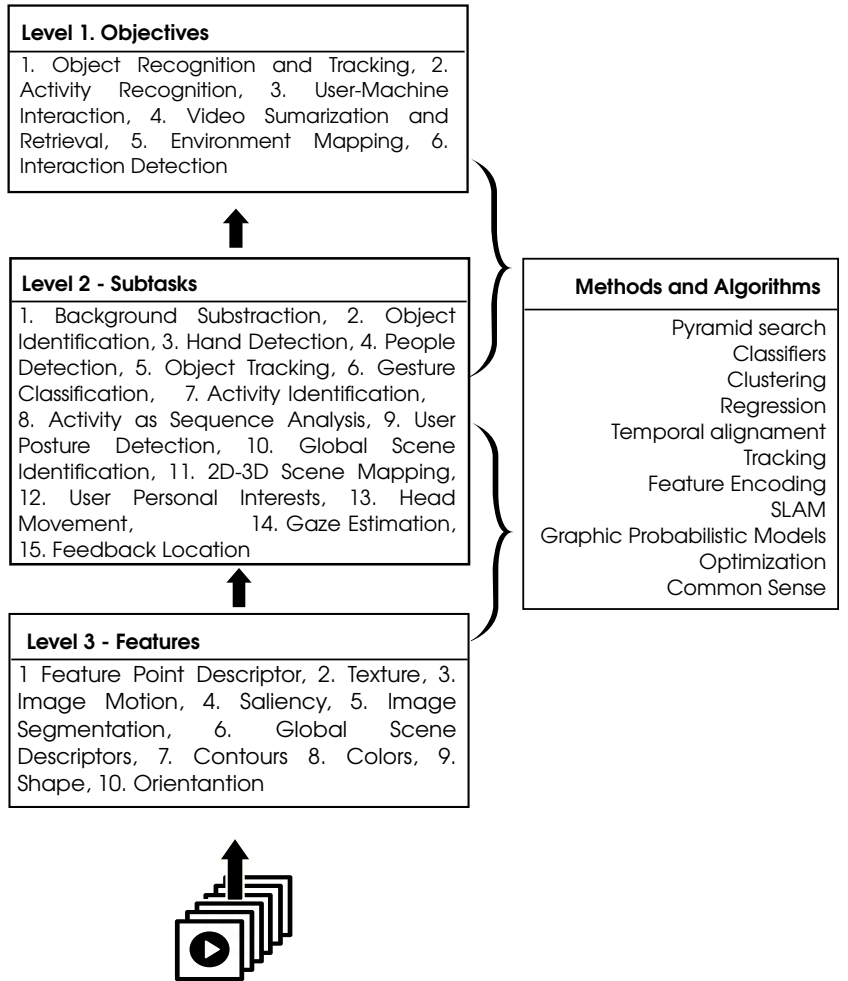

Fig. 4. Hierarchical structure to explain the state of the art in FPV video analysis.

addressed each of these 6 objectives. One important aspect is that some methods use multiple sensors within a data-fusion framework. For each objective, several examples of data-fusion and multi-sensor approaches are mentioned.

\subsubsection{Object recognition and tracking}

Object recognition and tracking is the most explored objective in FPV, and its results are commonly used as a starting point for more advanced tasks, such as activity recognition. Figure 5 summarizes some of the most important papers that focused on this objective.

In addition to the general opportunities and challenges of the FPV perspective, this objective introduces important aspects to be considered: i) Because of the uncontrolled characteristics of the videos, the number of objects, as well as their type, scale and point of view, are unknown $[27,77]$. ii) Active objects, as well as user's hands, are frequently occluded. iii) Because of the mobile nature of the wearable cameras, it is not easy to create background-foreground models. iv) The camera location makes it possible to build a priori information about the objects' position [27, 28].

Hands are among the most common objects in the user's field of view, and a proper detection, localization, and tracking could be a main input for other objectives. The authors in [28] highlight the difference between hand-detection and handsegmentation, particularly in the framework of wearable devices where the number of deployed computational resources, directly influences the battery life of the devices. In general, due to the hardware availability and price, hand-detection and 
TABLE 2

Summary of the articles reviewed in FPV video analysis according to the main objective

\begin{tabular}{|c|c|c|c|c|c|c|c|c|c|c|c|c|}
\hline & \multicolumn{6}{|c|}{ Objective } & \multicolumn{6}{|c|}{ Extra Categories } \\
\hline હ્ّ & 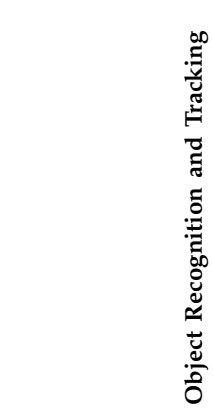 & 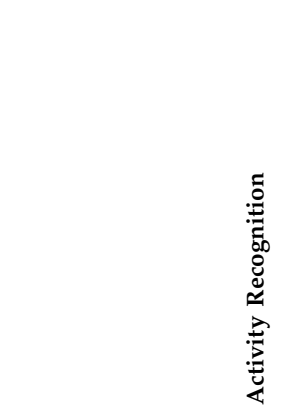 & 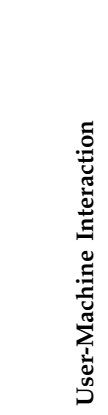 & 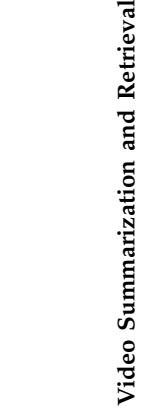 & 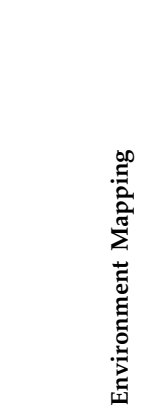 & 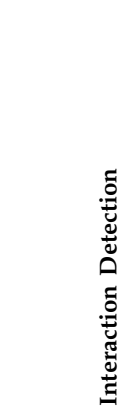 & 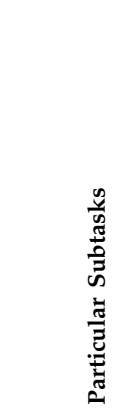 & 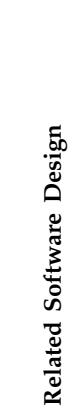 & 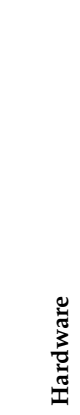 & 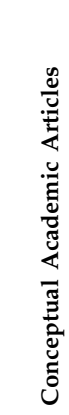 & 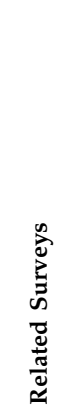 & 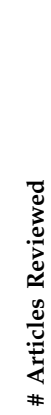 \\
\hline 1997 & & & & & & & & & [12] & & & 1 \\
\hline 1998 & {$[7,6,31]$} & [6] & & & & & & & [1] & & & 4 \\
\hline 1999 & {$[8,32]$} & {$[8,11]$} & [9] & & & & & & [9] & & & 4 \\
\hline 2000 & [33] & [34] & [35] & & & & & & [2] & & & 4 \\
\hline 2001 & [36] & & {$[37,38]$} & [39] & & & [21] & & & & & 5 \\
\hline 2002 & & & & {$[40,41]$} & & & & [41] & & & & 2 \\
\hline 2003 & & & [42] & {$[43,44]$} & & & & & & [14] & & 4 \\
\hline 2004 & & & {$[45,46]$} & {$[47]$} & & & & [47] & [48] & & & 4 \\
\hline 2005 & {$[49,50]$} & [49] & {$[51]$} & {$[52,53]$} & & & & [53] & [3] & & & 5 \\
\hline 2006 & & [5] & {$[54,55]$} & & & & & & {$[4,5]$} & & & 4 \\
\hline 2007 & {$[56,57]$} & [58] & & & {$[56,59,57]$} & & & & & & & 4 \\
\hline 2008 & [60] & & & & [60] & & & & & & & 1 \\
\hline 2009 & {$[27,50]$} & {$[61,62,63]$} & [64] & & & & & & & [17] & & 7 \\
\hline 2010 & [65] & {$[15,66]$} & & {$[66,67]$} & & & & & & & & 4 \\
\hline 2011 & {$[68,69]$} & {$[70,71]$} & & {$[72,70,73]$} & & & [74] & & [75] & & [19] & 9 \\
\hline 2012 & & {$[76,77,78,29,79,80]$} & [81] & & [82] & [23] & {$[83,84]$} & & {$[18]$} & & {$[18]$} & 12 \\
\hline 2013 & {$[85,86,30,87,88]$} & {$[89,90,91]$} & {$[13,92]$} & {$[24,91]$} & & [93] & [94] & & [22] & [95] & {$[20]$} & 16 \\
\hline 2014 & * & {$[96,97,98,99,100,101]$} & & $* *$ & & {$[102,103]$} & {$[28,104]$} & [105] & & [106] & & 27 \\
\hline
\end{tabular}

* $[107,108,109,110,111,112,113,114,16]$

** $[115,116,117,118,119,120]$

\begin{tabular}{|c|c|c|c|c|c|c|c|c|c|c|c|c|c|c|c|}
\hline \multicolumn{6}{|c|}{$\begin{array}{c}\text { [121] developes a pixel by pixel } \\
\text { classifier to locate human skin in } \\
\text { videos. }\end{array}$} & \multicolumn{6}{|c|}{$\begin{array}{l}\text { [49] proposes the first dataset for } \\
\text { object recognition in FPV. }\end{array}$} & \multicolumn{4}{|c|}{$\begin{array}{l}\text { [69] uses Multiple Instance Learning } \\
\text { to reduce the labeling requirements in } \\
\text { object recognition. }\end{array}$} \\
\hline 1997 & 1998 & 1999 & 2000 & 2001 & 2002 & 2003 & 2004 & 2005 & 2006 & 2007 & 2008 & 2009 & 2010 & 2012 & 2014 \\
\hline & \multicolumn{6}{|c|}{$\begin{array}{c}\text { [33] presents the "Augmented } \\
\text { Memory", following the ideas } \\
\text { presented in [9], as a killer application } \\
\text { in the field of FPV }\end{array}$} & \multicolumn{6}{|c|}{$\begin{array}{l}\text { [27] proposes a challenging dataset } \\
\text { of } 42 \text { daily use objects recorded at } \\
\text { different scales and perspectives. }\end{array}$} & \multicolumn{3}{|c|}{$\begin{array}{l}\text { [85] train a pool of models to dea } \\
\text { with changes in illumination. }\end{array}$} \\
\hline
\end{tabular}

Fig. 5. Some of the more important works in object recognition and tracking.

tracking is usually carried out using RGB videos. However, $[111,112]$ uses a chest-mounted RGB-D camera to improve the hand-detection and tracking performance in realistic scenarios. According to [49], hand detection could be divided into modeldriven and data-driven methods.

Model-driven methods search for the best matching configuration of a computational hand model (2D or $3 \mathrm{D})$ to recreate the image that is being shown in the video [122, 123, 124, 50, $111,112]$. These methods are able to infer detailed information of the hands, such as the posture, but in exchange large computational resources, highly controlled environments or extra sensors (e.g. Depth Cameras) could be required.
Data-driven methods use image features to detect and segment users' hands. The most commonly used features for this purpose are the color histograms looking to exploit the particular chromaticism of human skin, especially in suitable color spaces like HSV and YCbCr $[30,13,85,86]$. Color-based methods can be considered as the evolution of the pixel-by-pixel skin classifiers proposed in [121], in which color histograms are used to decide whether a pixel represents human skin. Despite their advantages, the color-based methods are far from being an optimal solution. Two of their more important restrictions are: i) The computational cost, because in each frame they have to solve the $O\left(n^{2}\right)$ problem implied by the pixel-by-pixel classification. ii) Their results highly influenced by significant changes 
in illumination, for example indoor and outdoor videos[28]. To reduce the computational cost, some authors suggest the use of superpixels $[13,30,86]$, however, an exhaustive comparison of the computational times of both approaches is still pending, and computationally efficient superpixel methods applied to video (especially FPV video) are still at an early stage [125]. Regarding the noisy results, the authors in [85, 13] train a pool of models and automatically select the most appropriate depending on the current environmental conditions.

In addition to hands, there is an uncountable number of objects that could appear in front of the user, whose proper identification could lead to development of some of the most promising applications of FPV. An example is "The Virtual Augmented Memory(VAM)" proposed by [33], where the device is able to identify objects, and to subsequently relate them to previous information, experiences or common knowledge available online. An interesting extension of the VAM is presented in [126], where the user is spatially located using his video, and is shown relevant information about the place or a particular event. In the same line of research, recent approaches have been trying to fuse information from multiple wearable cameras to recognize when the users are being recorded by a third person without permission. This is accomplished in [110, 127] using the motion of the wearable camera as the identity signature, which is subsequently matched in the third person videos without disclosing private information such as the face or the identity of the user.

The augmented memory is not the only application of object recognition. The authors in [77] develop an activity recognition method which based only a list of the used objects in the recorded video . Despite the importance of these applications, the problem of recognition is far from being solved due to the large amount of objects to be identified as well as the multiple positions and scales from which they could be observed. It is here that machine learning starts playing a key role in the field, offering tools to reduce the required knowledge about the objects [69] or exploiting web services (such as Amazon Turk) and automatic mining for training purposes [128, 29, 129, 58].

Once the objects are detected, it is possible to track their movements. In the case of the hands, some authors use the coordinates of center as the reference point [30], while others go a step further and use dynamic models [46, 55]. Dynamic models are widely studied and are successfully used to track hands, external objects $[59,56,59,60,57]$, or faces of other people [31].

\subsubsection{Activity recognition}

An intuitive step in the hierarchy of objectives is Activity Recognition, aimedat identifying what the user is doing in a particular video sequence. Figure 6 presents some of the most relevant papers on this topic. A common approach in activity recognition is to consider an activity as a sequence of events that can be modeled as Markov Chains or as Dynamic Bayesian Networks (DBNs) [6, 8, 34, 5, 63]. Despite the promising results of this approach, the main challenge to be solved is the scalability to multiple user and multiple strategies to solve a similar task.

Recently, two major methodological approaches for activity recognition are becoming popular: object based and motion based recognition. Object based methods aim to infer the activity using the objects appearing in video sequence [63, 71, 77], assuming of course that the activities can be described by the required group of objects( e.g. prepare a cup of coffee requires coffee, water and a spoon). This approach opens the door to highly scalable strategies based on web mining to know the objects usually required for different activities. However, after all, this approach depends on a proper Object Recognition step and on its own challenges (Section 2.1.1). Following an alternative path, during the last 3 years, some authors have been using the fact that different kind of activities create different body motions and as consequence different motion patterns in the video, for example: walking, running, jumping, skiing, reading, watching movies, among others [73, 80, 99]. It is remarkable the discriminative power of motion features for this kind of activities and the robustness to deal with the illumination and the color skin challenges.

Activity recognition is one of the fields that has drawn most benefits from the use of multiple sensors. This strategy started growing in popularity with the seminal work of Clarkson et al. $[34,32]$ where basic activities are identified using FPV video jointly with audio signals. An intuitive realization of the multisensor strategy allows to reduce the dependency between Activity Recognition and Object Recognition, by using RadioFrequency Identification (RFID) tags in the objects [58, 131, 132, 133]. However, the use of RFIDs reduces the applicability to environments previously tagged. The list of multiple sensors does not end with audio and RFIDs, it also contains Inertial Measurement Units [62], multiple sensors of the "SenseCam ${ }^{1 \text { " }}$ [70, 67], GPS [29], and eye-trackers [61, 78, 83, 74, 89].

\subsubsection{User-machine interaction}

As already mentioned, smart-glasses open the door to new ways for interaction between the user and his device. The device, being able to give feedback to the user, allows to close the interaction loop originated by the visual information captured and interpreted by the camera. Due to the scope of this paper, only approaches related to FPV video analysis are presented (we omit other sensors, such as audio and touch panels), categorizing them based on two approaches: i) the user sends information to the device, and ii) the device uses the information of the video to show the feedback to the user. Figure 7 shows some of the most important works concerning User-machine interaction.

In general, the interaction between the user and his device starts with intentional or unintentional command. An intentional command is a signal sent by the user using his hands through his camera. This kind of interaction is not a recent idea and several approaches have been proposed, particularly using static cameras $[135,136]$, which, as mentioned in section 2.1.1, can not be straightforwardly applied to FPV due to the mobile nature of wearable cameras. A traditional approach is to emulate the mouse of computers with the hands [124, 35, 37], allowing the user to point and click at virtual objects created in the head-up display. Other approaches look for more intuitive and technology focused ways of interaction. For example, the authors in [13] develop a gesture recognition algorithm to be used in an interactive museum using 5 different gestures:

1. Wearable device developed by Microsoft Research in Cambridge with accelerometers, thermometer, infrared and light sensor 


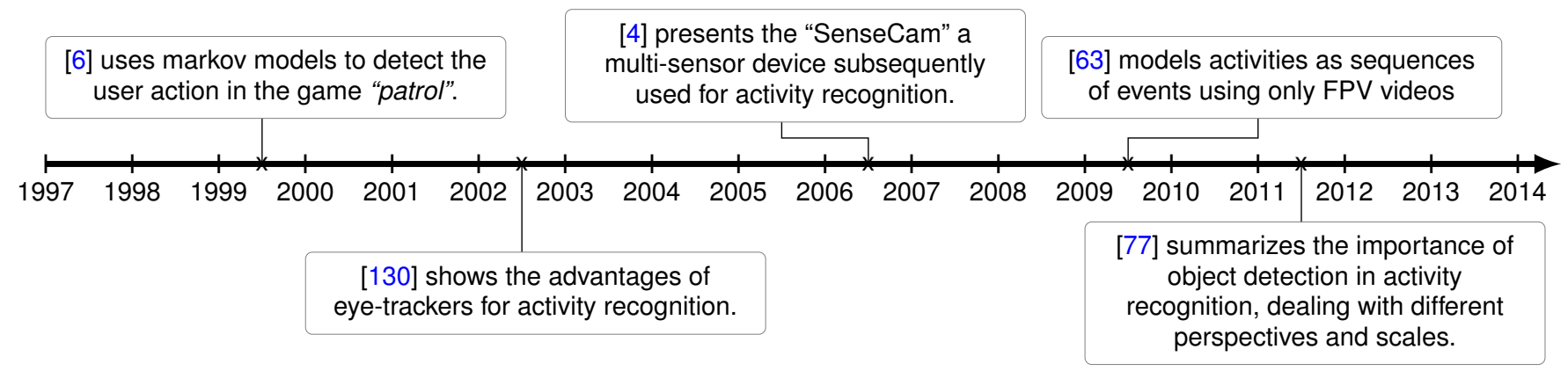

Fig. 6. Some of the more important works in activity recognition.

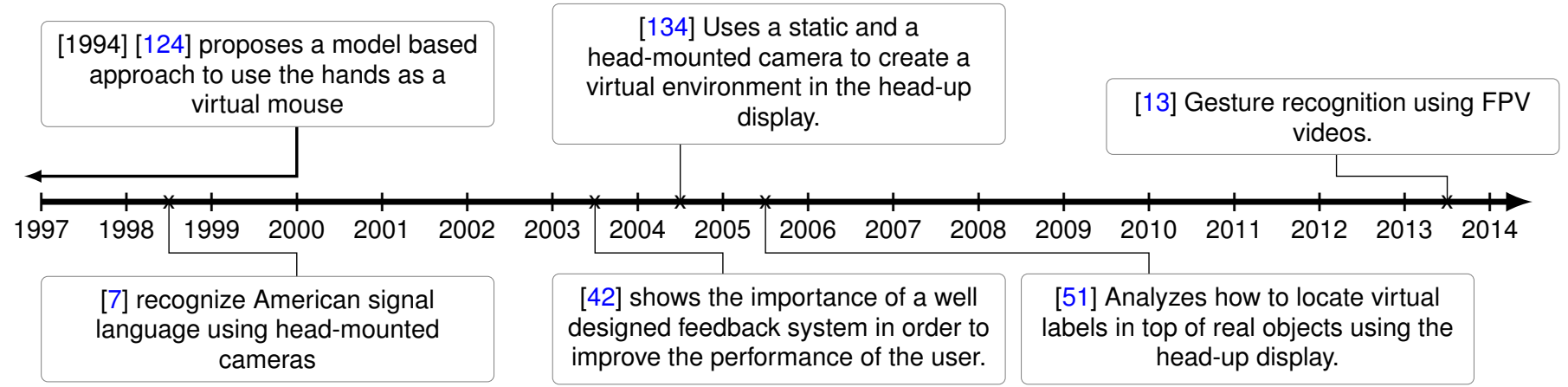

Fig. 7. Some of the more important works and commercial announcements in FPV.

"point out", "like", "dislike", "OK" and "victory". In [92], the head movements of the user are used to assist a robot in the task of finding a hidden object in a controlled environment. Under this perspective some authors combine static and wearable cameras[134, 7]. Quite remarkable are the results of Starner in 1998, being able to recognize American signal language with an efficiency of $98 \%$ with a static camera and head mounted camera. As is evident, hand-tracking methods can give important cues in this objective [137, 138, 139, 46], and make it possible to use features such as position, speed or acceleration of the users' hands.

Unintentional commands are triggers activated by the device using information about the user without his conscious intervention, for example: i) the user is cooking by following a particular recipe (Activity Recognition), and the device could monitor the time of different steps without the user previously asking for it. ii) The user is looking at a particular item [Object Recognition] in a store [GPS or Scene Recognition] then the device could show price comparisons and reviews. Unintentional commands could be detected using the results of other FPV objectives, the measurements of its sensors, or behavioural routines learned from the user while previously using his device, among others. From our point of view, these kinds of commands could be the next step of user-machine interaction for smart-glasses, and a main enabler to reduce the required time to interact with the device [95].

Regarding the second part of the interaction loop, it is important to properly design the feedback system to know when, where, how, and which information should be shown to the user. In order to accomplish this, several issues must be considered in order to avoid misbehaviour of the system that could work against the user's performance in addressing relevant tasks [42]. In this line, multiple studies develop methods to optimally locate virtual labels in the user's visual field, without occluding the important parts of the scene [64, 51, 81].

\subsubsection{Video summarization and retrieval}

The main task of Video summarization and retrieval is to create tools to explore and visualize the most important parts of large FPV video sequences [24]. The objective and main issue is perfectly summarized in [39] with the following sentence: "We want to record our entire life by video. However, the problem is how to handle such a huge data". In general, existing methods define importance functions to select the more relevant subsequences or frames of the video, and later cut or accelerate the less important ones [119]. Recent studies define the importance function using the objects appearing in the video [29], their temporal relationships and causalities [24], or as a similarity function, in terms of its composition, between them and intentional pictures taken with a traditional cameras [115]. A remarkable result is achieved in $[73,99]$ using motion features to segment videos according to the activity performed by the user. This work is a good example of how to take advantage of the camera movements in FPV, usually considered as a challenge, to achieve good classification rates.

The use of multiple sensors is common within this objective, and remarkable fusions have been made using brain measurements in [39, 40], gyroscopes, accelerometers, GPS, weather information and skin temperature in [43, 44, 52], and online available pictures in [115]. An alternative approach to video summarization is presented in [82] and [120], where multiple FPV videos of the same scene are unified using the collective attention of the wearable cameras as an importance function. In order to define whether the two videos recorded from different cameras are pointing at the same scene, the authors in [140] use superpixels and motion features to propose a 
similarity measurement. Finally, it is significant to mention that "Video summarization and retrieval" has led to important improvements in the design of the databases and visualization methods to store and explore the recorded videos [41, 47]. In particular, this kind of developments can be considered an important tool for reducing computational requirements in the devices, as well as alleviate privacy issues related with the place where videos are stored.

\subsubsection{Environment Mapping}

Environment Mapping aims at the construction of a 2D or 3D virtual representation of the environment surrounding the user. In general, the of variables to be mapped can be divided in two categories: physical variables, such as walls and object locations, and intangible variables, such as attention points. Physical mapping is the more explored of the two groups. It started to grow in popularity with [59], which showed how, by using multiple sensors, Kalman Filters and monoSLAM, it is possible to elaborate a virtual map of the environment. Subsequently, this method was improved by adding object identification and location as a preliminary stage [56, 57]. Physical mapping is one of the more complex tasks in FPV, particularly when $3 \mathrm{D}$ maps are required due to the calibration restrictions. This problem can be partially alleviated by using a multi-camera approach to infer the depth [60, 18]. Research on intangible variables, can be considered an emerging field in FPV. Existent approaches define attention points and attraction fields, mapping them in rooms with multiple people interacting [120].

\subsubsection{Interaction detection}

The objectives described above are mainly focused on the user of the device as the only person that matters in the scene. However, they hardly take into account the general situation in which the user is involved. We label the group of methods aiming to recognize the types of interaction that the user is having with other people as Interaction Detection. One of the main purposes in this objective is social interaction detection, as proposed by [23]. In their paper, the authors inferred the gaze of the other people and used it to recognize human interactions as monologues, discussions or dialogues. Another approach in this field was proposed by [93], which detected different behaviors of the people surrounding the user (e.g. hugging, punching, throwing objects, among others). Despite not being widely explored yet, this objective can be considered one of the most promising and innovative ones a in FPV due to the mobility and personalization capabilities of the coming devices.

\subsection{Subtasks}

As explained before, the proposed structure is based on objectives which are highly co-dependent. Moreover, it is common to find that the output of one objective is subsequently used as the input for the other (e.g. activity recognition usually depends on object recognition). For this reason, a common practice is to first address small subtasks, and later merge them to accomplish main objectives. Based on the literature review, we propose a total of 15 subtasks. Table 3 shows the number of articles analyzed in this survey that use a subtask (columns) in order to address a particular objective (rows). It is important to highlight the many-to-many relationship among objectives and subtasks, which means that a subtask could be used to address different objectives, and one objective could require multiple subtasks. To mention some: i) hand detection, as a subtask, could be the objective itself in object recognition, [30], but could also give important cues in activity recognition [78]; moreover, it could be the main input in the user-machine interaction [13]. ii) The authors in [77] performed object recognition to subsequently infer the performed activity. As we reckon that their names are self-explanatory, we omit separate explanation of each of the subtasks, with the possible exceptions of the following: i) Activity as a Sequence analyzes an activity as a set of ordered steps; ii) 2D-3D Scene Mapping builds a 2D or 3D virtual representation of the scene recorded; iii) User Personal Interests identifies the parts in the video sequence potentially interesting for the user using physiological signals such as brainwaves[40]; iv) Feedback location identifies the optimal place in the head-up display to locate the virtual feedback without interfering with the user's visual field.

As can be deduced from table 3, Hand detection plays an important role as the base for advanced objectives such as Object Recognition and User-Machine interaction. Global scene identification, as well as Object Identification, stand out as two important subtasks for activity recognition. More in detail, the tight bound between the Activity Recognition and the Object Recognition supports the idea of [77], which states that Activity Recognition is "all about objects". Moreover, the use of gaze estimation in multiple objectives confirms the advantages of the recent trend of using eye-trackers in conjunction with FPV videos. Finally, it can be noted that Background Subtraction has lost some of its reputation if compared with fixed camera scenarios, due to the highly unstable nature of the backgrounds when observed from the First-person perspective.

\subsection{Video and image features}

As mentioned before, FPV implies highly dynamic changes in the attributes and characteristics of the scene. Due to these changes, an appropriate selection of the features becomes critical in order to alleviate the challenges and exploit the advantages presented in section 2. As is well known, feature selection is not a trivial task, and usually implies an exhaustive search in the literature and extensive testing to identify which method leads to optimal results.

The process of feature extraction is carried out at different levels, starting from the pixel level, with color channels of the image, and subsequently extracting more elaborated indicators at the frame level, such as saliency, texture, superpixels, gradients, etc. As expected, these features can be used to address some of the subtasks, such as object recognition or scene identification. However, they do not include any kind of dynamic information. To add dynamic information in the analysis, different approaches can be followed, for example analyzing the geometrical transformation between two frames to obtain image Motion features such as optical flow, or aggregating frame level features in temporal windows. Usually, dynamic features tend to be computationally expensive, and are therefore usually applied to objectives in which the video is processed once the activities have finished. Particularly interesting is the method presented in [125], which uses the information of the superpixels of the previous frame to initialize and compute the current 
TABLE 3

Number of times that a subtask is performed to accomplish a specific objective

\begin{tabular}{|c|c|c|c|c|c|c|c|c|c|c|c|c|c|c|c|}
\hline Objective & 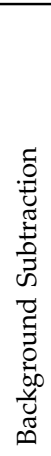 & 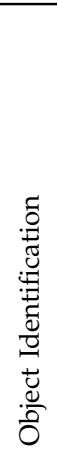 & 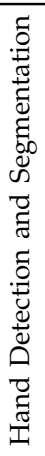 & 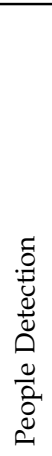 & 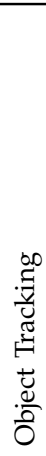 & 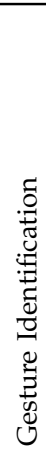 & 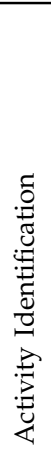 & 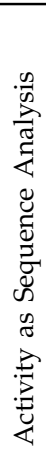 & 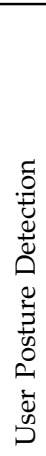 & 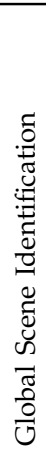 & 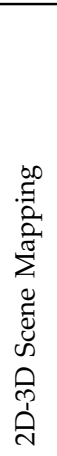 & 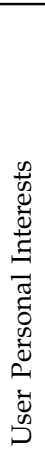 & 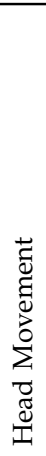 & 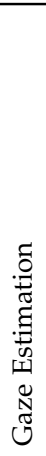 & 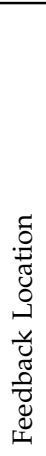 \\
\hline Object Recognition and Tracking & 4 & 15 & 13 & 3 & 10 & & & & & 2 & & & 1 & 1 & \\
\hline Activity Recognition & 3 & 8 & 3 & 1 & & & 13 & 2 & 1 & 8 & 1 & & 6 & 6 & \\
\hline User-Machine Interaction & & & 6 & & 3 & 2 & & & & & & & 1 & & 3 \\
\hline Video Summarization & 1 & 4 & 1 & 4 & & & 5 & 1 & & 4 & & 1 & 2 & 1 & \\
\hline Environment Mapping & & 3 & & & 4 & & & & & & 5 & & & 1 & \\
\hline Interaction Detection & & & & 2 & 1 & & 2 & & & & 1 & & 2 & 2 & \\
\hline
\end{tabular}

frame superpixels, thus reducing the computational complexity of the algorithm by $60 \%$.

Table 4 shows the most commonly used features in FPV to address a particular subtask. The features are listed in the rows and the subtasks in the columns. Note that color histograms are by far the most commonly used feature for almost all the subtasks, despite being highly criticized due to their dependence on illumination changes. Another group of features frequently used for several subtasks is Image Motion. Some of its most remarkable results are for Activity Recognition in [73, 99], for Video Summarization in [119], and recently as the input of a Convolutional Neural Network (CNN) to create a biometric sensor that is able to identify the user recording the video in [127]. The use of Feature Point Descriptors (FPD) is also worth noting. As expected, they are popular for object identification, but it is also remarkable their application to identify relevant places such as touristic hotspots $[72,15,66]$. Note from the table that the "dynamic objectives" like Activity Recognition and Video Summarization are the ones which take the most advantage of the Motion features, while Object Recognition is mainly based on frame features such as FPD and Color histograms.

From our previous studies in Hand-detection and Handsegmentation using multiple features and superpixels, we want to point out that Color features are a good approach, particularly if a suitable color space is exploited [30]. We found that low level features such as Color Histograms could help to reduce the computational complexity of the methods and get close to real time applications. On the other side, under large illumination changes, in [28] we highlight how Colorbased hand-segmentators could introduce and disseminate in the system noise created by hands missdetections. To alleviate this problem, we used shape features, such as HOG, in order to pre-filter wrong measurements and improve the classification rate of the overall system.

The two empty columns in table 4 can be explained as follows: Activity as a sequence is usually chained with the output of a short activity identification [11, 61, 72], whereas identification of the User Posture is accomplished in [5] without employing visual features, but using GPS and accelerometers.

\subsection{Methods and algorithms}

Once that features are selected and estimated, the next step is to use them as inputs to reach the objective (outputs). At this point, quantitative methods start playing the main role, and as expected, an appropriate selection directly influences the quality of the results, ultimately showing whether the advantages of the FPV perspective are being exploited, or whether the FPVrelated challenges are impacting the objectives negatively. Table 5 shows the number of occurrences of each method (rows) being used to accomplish a particular objective or a subtask (columns).

The table highlights classifiers as the most popular tool in FPV, which is commonly used to assign a category to an array of characteristics (see [141] for a more detailed survey on classifiers). The use of classifiers is wide and varies from general applications, such as scene recognition [69], to more specific, such as activity recognition given a set of objects [78]. Particularly, we found that the most used are the Support Vector Machines (SVM) due to their capability to deal with non-separable non-linear multi-label problems using low computational resources. On the other hand, SVMs require large labeled training sets which restricts the range of potential applications.

In our previous works we performed a comparison of the performance of multiple features (HOG, GIST, Color) and classifiers (SVM, Random Forest, Random Threes) to solve the hand-detection problem [28]. Our conclusion was that HOGSVM was the best performing combination, achieving a classification rate of $90 \%$ and $93 \%$ of true positives and true negatives respectively. Another group of methods commonly used are clustering algorithms due to its simplicity, computational cost, and small requirements in the training datasets. Despite their advantages, clustering algorithms could require post-processing 
TABLE 4

Number of times that each feature is used in to solve an objective or subtask

\begin{tabular}{|c|c|c|c|c|c|c|c|c|c|c|c|c|c|c|c|c|c|c|c|c|c|c|}
\hline & & \multicolumn{6}{|c|}{ Objectives } & \multicolumn{15}{|c|}{ Subtasks } \\
\hline & & 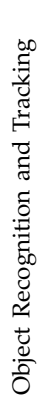 & 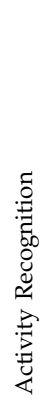 & 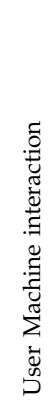 & 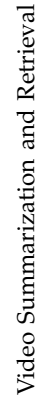 & 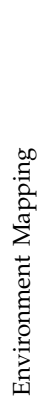 & 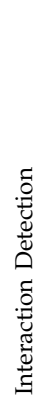 & 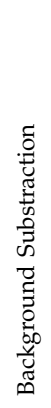 & 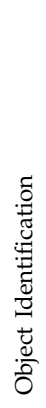 & 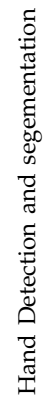 & 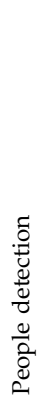 & 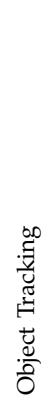 & 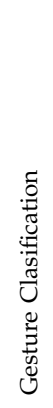 & 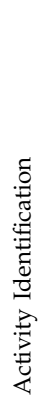 & 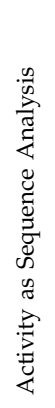 & 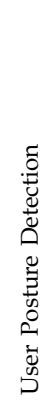 & 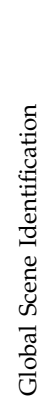 & 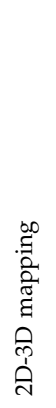 & 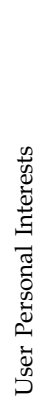 & 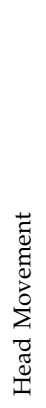 & 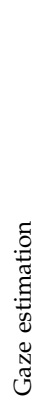 & 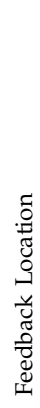 \\
\hline \multirow{8}{*}{ FPD } & SIFT & 9 & 5 & 1 & 4 & 3 & & 2 & 14 & & 1 & & & 1 & & & 2 & & & & & \\
\hline & GFTT & 1 & 1 & & & & & & & & & 1 & & & & & & & & & & \\
\hline & BRIEF & 2 & & & & & & & & 1 & & & & & & & 1 & & & & & \\
\hline & FAST & 1 & & & & & & & & & & & & & & & 1 & & & & & \\
\hline & SURF & 2 & 6 & & 1 & & & & 2 & & 1 & 1 & & 2 & & & 2 & & & & & \\
\hline & Diff. of Gaussians & & & & 1 & & & & 1 & & & & & & & & & & & & & \\
\hline & ORB & 1 & & & & & & & & 1 & & & & & & & & & & & & \\
\hline & STIP & & 2 & & & & & 1 & 1 & & & & & & & & & & & & & \\
\hline \multirow{5}{*}{ Texture } & Wiccest & & & & 1 & & & & & & & & & 1 & & & & & & & & \\
\hline & Laplacian Transform & 1 & & & & & & & & 1 & & & & & & & & & & & & \\
\hline & Edge Histogram & & 1 & 1 & 1 & & & & 1 & & & & & & & & & & 1 & & & 1 \\
\hline & Wavlets & 1 & & & & & & & & & 1 & & & & & & & & & & & \\
\hline & Other & & 1 & & 1 & & & & & & & & & 1 & & & & & & & & \\
\hline \multirow{3}{*}{ Saliency } & GBVS & 1 & 1 & & & & & & & & & & & & & & & & & & 4 & \\
\hline & Other & & 1 & & & & & & 1 & & & & & & & & & & & & 1 & \\
\hline & MSSS & & & 1 & & & & & & & & & & & & & & & & & & 1 \\
\hline \multirow{3}{*}{ Motion } & Optical Flow & 5 & 14 & 2 & 5 & & 1 & 5 & 1 & 2 & & 2 & & 6 & & & 1 & & & 4 & 5 & \\
\hline & Motion Vectors & & 1 & 1 & 3 & & & & & & & 1 & & & & & 1 & & 1 & 3 & 1 & \\
\hline & Temporal Templates & & 1 & & & & & & & 1 & & & & & & & & & & & & \\
\hline \multirow{2}{*}{ Glob. Scene } & CRFH & & 1 & & & & & & & & & & & & & & 1 & & & & & \\
\hline & GIST & 1 & 2 & & & & & & & 1 & & & & & & & 2 & & & & 1 & \\
\hline \multirow{2}{*}{ Img. Segment. } & Superpixels & 2 & 2 & 1 & & & & & 2 & 3 & & & & & & & & & & & & \\
\hline & Blobs & 2 & & & & & & & & 1 & 1 & & & & & & & & & & & \\
\hline Contour & OWT-UCM & 1 & 3 & & & & & 2 & 2 & & & & & & & & & & & & & \\
\hline Color & Histograms & 21 & 20 & 11 & 10 & & & 3 & 8 & 20 & 4 & 4 & 1 & 5 & & & 7 & & 1 & & 2 & \\
\hline Shapes & HOG & 6 & 4 & & 3 & & 1 & & 2 & 5 & 1 & 1 & & 3 & & & 1 & & & 1 & & \\
\hline Orientation & Gabor & & & & 1 & & & & & & & & & 1 & & & & & & & & \\
\hline
\end{tabular}

analysis of the results in order to endow them with human interpretation.

Another promising group of tools are the Probabilistic Graphical Models (PGMs), which can be interpreted as a framework to combine multiple sensors and chain results from different methods in a unique probabilistic hierarchical structure (e.g. to recognize the object and subsequently use it to infer the activity). Dynamic Bayesian Networks (DBNs) are a particular type of PGMs which include time in their structure, in turn making them suitable for application in video analysis [142]. As an example, DBNs are frequently used to represent activities as sequences of events $[6,8,34,5,63]$. It is common to find that particular methods, such as Dirichlet Process Mixture Models
(DPMM), are presented in their PGM notation, however given the promising recent results achieved in Activity Recognition and Video Segmentation, we decided to group them separately.

As stated in section 2.3, there is a large number of features that can be extracted for FPV applications. A common practice is to mix or chain multiple features before using them as input of a particular algorithm (table 5). This practice usually results in extremely large vectors of features that can lead to computationally expensive algorithms. In this context, the role of Feature Encoding methods, such as Bag-of-Words, is crucial to control the size of the inputs. We highlight the importance that some authors are giving to this tool, which, despite not being an automatic strategy like Linear Discriminant Analysis (LDA) 
TABLE 5

Mathematical and computational methods used in objective or each subtask

\begin{tabular}{|c|c|c|c|c|c|c|c|c|c|c|c|c|c|c|c|c|c|c|c|c|c|}
\hline & \multicolumn{6}{|c|}{ Objective } & \multicolumn{15}{|c|}{ Subtasks } \\
\hline & 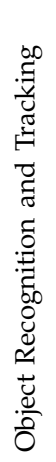 & 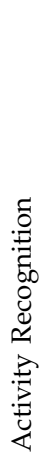 & 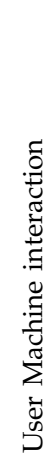 & 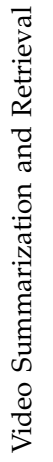 & 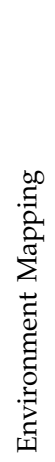 & 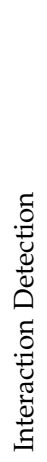 & 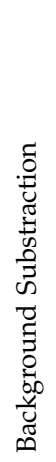 & 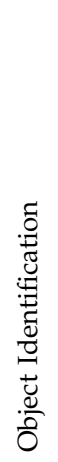 & 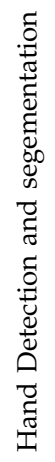 & 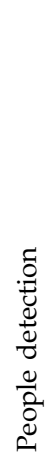 & 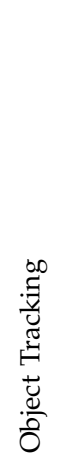 & 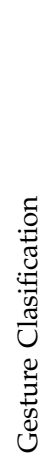 & 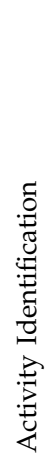 & 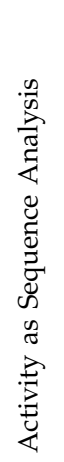 & 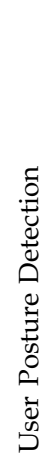 & 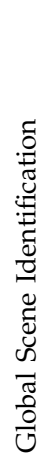 & 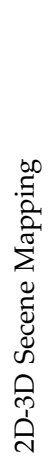 & 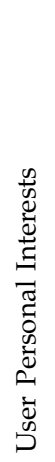 & 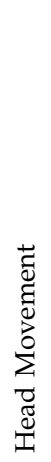 & 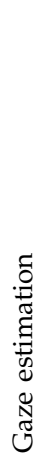 & 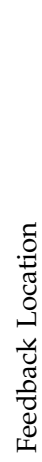 \\
\hline 3D Mapping & 3 & 1 & & & 4 & & & & & & & & & & & & 5 & & & & \\
\hline Classifiers & 21 & 29 & 3 & 9 & 1 & 2 & 3 & 17 & 15 & 2 & & 2 & 15 & & & 4 & & 1 & 2 & 1 & \\
\hline Clustering & 4 & 8 & 3 & 5 & & 2 & & 3 & 6 & & & & 3 & & & 8 & & & & 1 & \\
\hline Comon sense & 3 & 8 & & & & 1 & & 3 & 3 & & & & 2 & & & & 1 & & & 3 & \\
\hline DPMM & & 1 & & 2 & & & & & & & & & 3 & & & & & & & & \\
\hline Feature Encoding & 4 & 6 & & 3 & & & & 6 & & & & & 3 & & & 3 & & & & & \\
\hline Optimization & & 1 & 1 & 1 & & & & & & & & & 1 & & & & & & & & 1 \\
\hline PGM & 6 & 17 & & 3 & & 3 & 2 & 1 & & & & 1 & 11 & 1 & & 6 & & & 1 & 7 & \\
\hline Pyramid Search & 4 & 4 & & & & 1 & & 3 & 2 & 2 & & & 2 & & & & & & & & \\
\hline Regresions & 1 & 1 & & & 1 & & & & & 1 & & & & & & 1 & 1 & & 1 & 1 & \\
\hline Temporal Alignament & & & & 1 & & & & & & & & & & 1 & & & & & & & \\
\hline Tracking & 4 & & 3 & & 5 & & & & & & 7 & & & & & & 1 & & 2 & & \\
\hline
\end{tabular}

and Principal Components Analysis (PCA), can nevertheless help to include human intuition in the analysis. As an example, the authors in [97] use BoW in Activity Recognition taking into account the presence, level of attention, and the role of the objects in the video.

The use of machine learning methods (e.g. classifiers, clustering, regressors) introduces an important question to the analysis: how to train the algorithms on realistic data without restricting their applicability? This question is widely studied in the field of Artificial Intelligence, and two different approaches are commonly followed, namely unsupervised and supervised learning [143]. Unsupervised learning requires less human interaction in training steps, but requires human interpretation of the results. Additionally, unsupervised methods have the advantage of being easily adaptable to changes in the video (e.g. new objects in the scene or uncontrolled environments [62]). The most commonly used unsupervised method in FPV are the clustering algorithms, such as k-means. In fact, the best performing superpixels are the result of an unsupervised clustering procedure applied over a raw image[144]. In [125] we proposed an optimization of the SLIC superpixels, and latter in [145] we introduced a new superpixel method based on Neural Networks. The proposed algorithm is a self-growing map that adapts its topology to the frame structure taking advantage of the dynamic information available in the previous frames.

Regarding the supervised methods, their results are easily interpretable but commonly imply higher requirements in the training stage. As an example, at the beginning of this section we highlighted some of the applications of SVMs. Supervised methods use a set of inputs, previously labeled, to parametrize the models. Once the method is trained, it can be used on new instances without any additional human supervision. In general, supervised methods are more dependent on the training data, fact which could work against their performance when used on newly-introduced cases [77, 24, 62, 23, 58, 29, 146]. In order to reduce the training requirements, and take advantage of the useful information available on Internet, some authors create their datasets using services like Amazon Mechanical Turk [128, 29], automatic web mining [129, 58], or image repositories [115]. We named this practice in table 5 as Common Sense.

Weakly supervised learning is another commonly used strategy, considered as a middle point between supervised and unsupervised learning. This strategy is used to improve the supervised methods in two aspects: i) extending the capability of the method to deal with unexpected data; and ii) reducing the necessity for large training datasets. Following this trend, the authors of $[66,15]$ used Bag of Features (BoF) to monitor the activity of people with dementia. Later, $[69,71]$ used Multiple Instance Learning (MIL) to recognize objects using general categories. Afterwards, [72] used BoF and Vector of Locally Aggregated Descriptors (VLAD) to temporally align a sequence of videos. Eventually, let us mention Deep learning, a relatively recent approach which combines supervised and unsupervised learning techniques in a unified framework, where low level significant features are learned in an unsupervised fashion 
[147].

\section{Public datasets}

In order to support their results and create benchmarks in FPV video analysis, some authors have provided their datasets for public use to the academic community. The first publicly available FPV dataset is released by [49]. It consists of a video containing 600 frames recorded in a controlled office environment using a camera on the left shoulder, while the user interacts with five different objects. Later, [27] proposed a larger dataset with two people interacting with 42 object instances. The latter one is commonly considered as the first challenging FPV dataset because it guaranteed the requirements identified by [9]: i) Scale and texture variations, ii) Frame resolution, iii) Motion blur, and iv) Occlusion by hand.

Implicitly, previous sections explain some of the main characteristics of FPV videos. In [148], these characteristics are compared for several FPV and Third Person Vision (TPV) datasets and their classification capabilities are evaluated. The authors reach a classification accuracy of $80.9 \%$ using blur, illumination changes, and optical flow as input features. In their study they also found a considerable difference in the classification rate explained by the camera position. The authors concluded that the more stable the camera, the less blur and motion and then the less discriminative power of these features. We highlight this difference as an important finding because it opens the door to an interesting discussion concerning which kind of videos, based on quantitative measurements, should be considered as FPV. Extra evidence about the role of the non-wearable cameras, such as hand-held devices when they are used to record from a first person perspective, is still pending. Our intuition points that, despite having some of the challenging characteristics of wearable cameras like mobile backgrounds and unstable motion patterns, hand-held videos would drastically differ in terms of features compared in [148].

Table 6 presents a list of the publicly-available datasets, along with their characteristics. Of particular interest are the changes in the camera location, which have evolved from shoulderbased to the head-based. These changes are clearly explained by the trend of the smart-glasses and action cameras (see Table 1). Also noticeable are the changes in the objectives of the datasets, moving from low level, such as object recognition, to more complex objectives, such as social interaction and usermachine interaction. It should also be noted that less controlled environments have recently been proposed to improve the robustness of the methods in realistic situations. In order to highlight the robustness of their methods, several authors evaluated them on Youtube sequences recorded using goPro cameras [73].

Another aspect to highlight from the table is the availability of multiple sensors in some of the datasets. For instance, the Kitchen dataset [62] includes four sensors, the GTEA approach [78] includes eye tracking measurements, and the Egocentric Intel/Creative [111] was recorded with a RGBD camera.

\section{CONCLUSION AND FUtURE RESEARCH}

Wearable devices such as smart-glasses will presumably constitute a significant share of the technology market during the coming years, bringing new challenges and opportunities in video analytics. The interest in the academic world has been growing in order to satisfy the methodological requirements of this emerging technology. This survey provides a summary of the state of the art from the academic and commercial point of view, and summarizes the hierarchical structure of the existent methods. This paper shows the large number of developments in the field during the last 20 years, highlighting main achievements and some of the up-coming lines of study.

From the commercial and regulatory point of view, important issues must be faced before the proper commercialization of this new technology can take place. Nowadays, the privacy of the recorded people is one of the most discussed ones, as these kinds of devices are commonly perceived as intruders [17]. Other important aspects are the legal regulations depending on the country, , and the intention of the user to avoid recording private places or activities[113]. Another hot topic is the real applicability of smart-glasses as a massive consumption device or as a task-oriented tool to be worn only in particular scenarios. In this field, the technological companies are designing their strategies in order to reach out to specific markets. As an illustration, recent turn of events has seen Google move out of the glass project (originally intended to end with a massively commercialized product), in order to target the enterprise market. Microsoft, on the other hand, recently announced its task-oriented holographic device "HoloLens" embodied with a larger array of sensors.

From the academic point of view, the research opportunities in FPV are still wide. Under the light of this bibliographic review and our personal experience, we identify 4 main hot topics:

- Existing methods are proposed and executed in previously recorded videos. However, none of them seems to be able to work in a closed-loop fashion, by continuously learning from users' experiences and adapt to the highly variable and uncontrollable surrounding environment. From our previous studies [149, 150], we believe that a cognitive perspective could give important cues to this aspect and could aid the development of the self-adaptive devices.

- The personalization capabilities of smart-glasses open the door to new learning strategies. Incoming methods should be able to receive personalized training from the owner of the device. We have found out, for instance, that this kind of approach can help alleviate problems, such as changes in the color skin models from different users [30] in a hand detection application. Indeed, color features, as stressed in 4 , has proven to be extremely suitable to be exploited in this field.

- This survey focuses on methods for addressing tasks accomplished mainly by one user coupled with a single wearable device. However, cooperative devices would be useful to increase the number of applications in areas such as environment mapping, military applications, cooperative games, sports, etc.

- Finally, regarding the real time requirements, important developments should be made in order to optimally compute FPV methods without draining the battery. This must be accomplished both from the hardware and the software side. On the one hand, progress still needs to be made on the processing units of the devices. On the other, lighter, faster and better optimized methods are yet to be designed and tested. Our personal experience lead us to explore 
TABLE 6

Current datasets and sensors availability

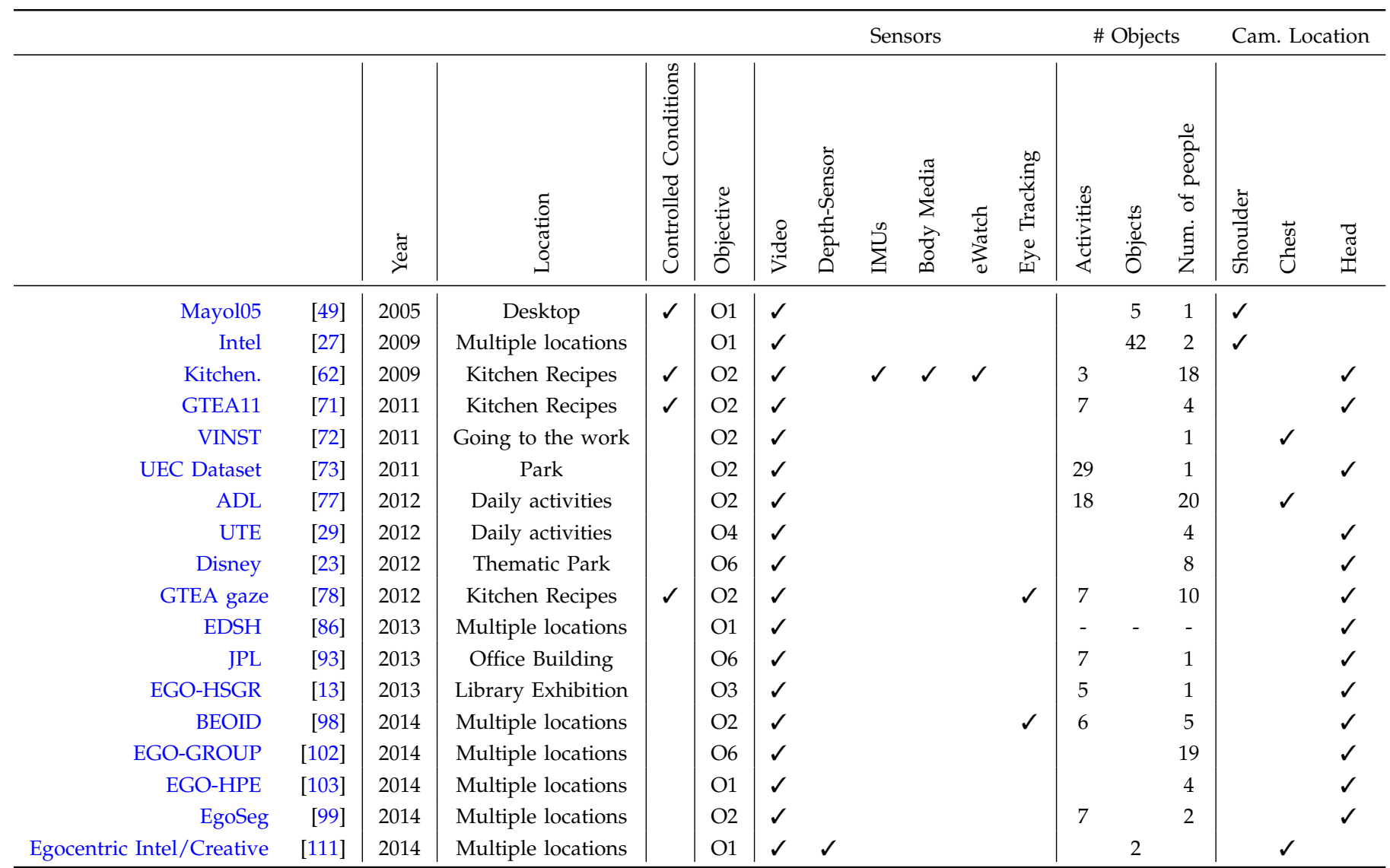

* Objectives: [O1] Object Recognition and Tracking. [O2] Activity Recognition. [O3] User-Machine Interaction. [O4] Video Summarization. [O5] Phisical Scene Reconstruction. [O6] Interaction Detection.

${ }^{* *}$ The table summarizes the characteristic described in the technical reports or the papers proposing the datasets.

fast machine learning methods [28] for hand detection, in the trend highlighted by table 5 , and to discard standard features such as optic flow [30] because of computational restrictions. Promising methods in standard computer vision research, such as superpixel methods, were built from scratch in [145] in order to make them faster and better suited for video analysis [125]. Eventually, important cues to the problem of computational power optimization may also be found in cloud computing and high performance computing.

\section{REFERENCES}

[1] S. Mann, “"WearCam” (Wearable Camera): Personal Imaging Systems for Long-term Use in Wearable Tetherless Computer-mediated Reality and Personal Photo/videographic Memory Prosthesis," in Wearable Computers, (Pittsburgh), pp. 124-131, IEEE Computer Society, 1998.

[2] W. Mayol, B. Tordoff, and D. Murray, "Wearable Visual Robots," in International Symposium on Wearable Computers, (Atlanta), pp. 95-102, IEEE Computer Society, 2000.

[3] W. Mayol, A. Davison, B. Tordoff, and D. Murray, "Applying Active Vision and SLAM to Wearables," in Springer Tracts in Advanced Robotics (P. Dario and R. Chatila, eds.), vol. 15 of Springer Tracts in Advanced Robotics, pp. 325334, Berlin, Heidelberg: Springer Berlin Heidelberg, 2005

[4] S. Hodges, L. Williams, E. Berry, S. Izadi, J. Srinivasan, A. Butler, G. Smyth, N. Kapur, and K. Wood, "Sensecam: a Retrospective Memory Aid," in International Conference of Ubiquitous Computing, pp. 177-193, Springer Verlag, 2006.
[5] M. Blum, A. Pentland, and G. Tröster, "InSense : Life Logging," MultiMedia, vol. 13, no. 4, pp. 40-48, 2006.

[6] T. Starner, B. Schiele, and A. Pentland, "Visual Contextual Awareness in Wearable Computing," in International Symposium on Wearable Computers, pp. 50-57, IEEE Computer Society, 1998.

[7] T. Starner, J. Weaver, and A. Pentland, "Real-Time American Sign Language Recognition Using Desk and Wearable Computer Based Video," Transactions on Pattern Analysis and Machine Intelligence, vol. 20, no. 12, pp. 1371-1375, 1998

[8] B. Schiele, T. Starner, and B. Rhodes, "Situation Aware Computing with Wearable Computers," in Augmented Reality and Wearable Computers, pp. 120, 1999

[9] B. Schiele, N. Oliver, T. Jebara, and A. Pentland, “An Interactive Computer Vision System DyPERS: Dynamic Personal Enhanced Reality System," in Computer Vision Systems, vol. 1542 of Lecture Notes in Computer Science, pp. 51-65, Berlin, Heidelberg: Springer Berlin Heidelberg, Sept. 1999.

[10] T. Starner, Wearable Computing and Contextual Awareness. PhD thesis, Massachusetts Institute of Technology, 1999.

[11] H. Aoki, B. Schiele, and A. Pentland, "Realtime Personal Positioning System for a Wearable Computer," in Wearable Computers, (San Francisco, CA, USA), pp. 37-43, IEEE Comput. Soc, 1999.

[12] S. Mann, "Wearable Computing: a First Step Toward Personal Imaging," Computer, vol. 30, no. 2, pp. 25-32, 1997.

[13] G. Serra, M. Camurri, and L. Baraldi, "Hand Segmentation for Gesture Recognition in Ego-vision," in Workshop on Interactive Multimedia on Mobile $\mathcal{E}$ Portable Devices, (New York, NY, USA), pp. 31-36, ACM Press, 2013.

[14] S. Mann, J. Nolan, and B. Wellman, "Sousveillance: Inventing and Using Wearable Computing Devices for Data Collection in Surveillance Environments.," Surveillance E Society, vol. 1, no. 3, pp. 331-355, 2003. 
[15] S. Karaman, J. Benois-Pineau, R. Megret, V. Dovgalecs, J. Dartigues, and Y. Gaestel, "Human Daily Activities Indexing in Videos from Wearable Cameras for Monitoring of Patients with Dementia Diseases," in International Conference on Pattern Recognition, pp. 4113-4116, Ieee, Aug. 2010.

[16] K. Liu, S. Hsu, and C. Huang, "First-person-vision-based Driver Assistance System," in Audio, Language and Image Processing, pp. 4-9, 2014.

[17] D. Nguyen, G. Marcu, G. Hayes, K. Truong, J. Scott, M. Langheinrich, and C. Roduner, "Encountering Sensecam: Personal Recording Technologies in Everyday Life," in International Conference on Ubiquitous Computing, 2009.

[18] T. Kanade and M. Hebert, "First-person Vision," Proceedings of the IEEE, vol. 100, pp. 2442-2453, Aug. 2012.

[19] D. Guan, W. Yuan, A. Jehad-Sarkar, T. Ma, and Y. Lee, "Review of Sensorbased Activity Recognition Systems," IETE Technical Review, vol. 28, no. 5, p. $418,2011$.

[20] A. Doherty, S. Hodges, and A. King, "Wearable Cameras in Health," American Journal of Preventive Medicine, vol. 44, no. 3, pp. 320-323, 2013.

[21] M. Land and M. Hayhoe, "In What Ways Do Eye Movements Contribute to Everyday Activities?," Vision research, vol. 41, pp. 3559-65, Jan. 2001.

[22] S. Mann, M. Ali, R. Lo, and H. Wu, "Freeglass for Developers,haccessibility, and Ar Glass+ Lifeglogging Research in a (Sur/sous) Veillance Society," in Information Society, pp. 51-56, 2013.

[23] A. Fathi, J. Hodgins, and J. Rehg, "Social Interactions: A First-Person Perspective," in Computer Vision and Pattern Recognition, (Providence, RI) pp. 1226-1233, IEEE, June 2012.

[24] Z. Lu and K. Grauman, "Story-Driven Summarization for Egocentric Video," in Computer Vision and Pattern Recognition, (Portland, OR, USA), pp. 2714-2721, IEEE, June 2013.

[25] A. Yarbus, Eye Movements and Vision. New York, New York, USA: Plenum Press, 1967.

[26] I. Bisio, A. Delfino, F. Lavagetto, and M. Marchese, "Opportunistic detection methods for emotion-aware smartphone applications," Creating Personal, Social, and Urban Awareness Through Pervasive Computing, p. 53, 2013.

[27] M. Philipose, "Egocentric Recognition of Handled Objects: Benchmark and Analysis," in Computer Vision and Pattern Recognition, (Miami, FL), pp. 1-8, IEEE, June 2009

[28] A. Betancourt, "A Sequential Classifier for Hand Detection in the Framework of Egocentric Vision," in Conference on Computer Vision and Pattern Recognition Workshops, vol. 1, (Columbus, Ohio), pp. 600-605, IEEE, June 2014.

[29] J. Ghosh and K. Grauman, "Discovering Important People and Objects for Egocentric Video Summarization," in Computer Vision and Pattern Recognition, pp. 1346-1353, IEEE, June 2012.

[30] P. Morerio, L. Marcenaro, and C. Regazzoni, "Hand Detection in First Person Vision," in Information Fusion, (Istanbul), pp. 1502 - 1507, University of Genoa, 2013.

[31] G. Bradski, "Real Time Face and Object Tracking as a Component of a Perceptual User Interface," in Applications of Computer Vision, pp. 14-19, IEEE, 1998.

[32] B. Clarkson and A. Pentland, "Unsupervised Clustering of Ambulatory Audio and Video," in Acoustics, Speech, and Signal Processing, (Phoenix, AZ), pp. 1520-6149, IEEE, 1999.

[33] J. Farringdon and V. Oni, "Visual Augmented Memory," in International Symposium on wearable computers, (Atlanta GA), pp. 167-168, 2000.

[34] B. Clarkson, K. Mase, and A. Pentland, "Recognizing User Context Via Wearable Wensors," in Digest of Papers. Fourth International Symposium on Wearable Computers, (Atlanta, GA, USA), pp. 69-75, IEEE Comput. Soc, 2000.

[35] T. Kurata, T. Okuma, M. Kourogi, and K. Sakaue, "The Hand-mouse: A Human Interface Suitable for Augmented Reality Environments Enabled by Visual Wearables," in Symposium on Mixed Reality, (Yokohama), pp. 188$189,2000$.

[36] T. Kurata, T. Okuma, and M. Kourogi, "Vizwear: Toward Human-centered Interaction Through Wearable Vision and Visualization," Lecture Notes in Computer Science, vol. 2195, no. 1, pp. 40-47, 2001.

[37] T. Kurata and T. Okuma, "The Hand Mouse: Gmm Hand-color Classification and Mean Shift Tracking," in Recognition, Analysis, and Tracking of Faces and Gestures in Real-Time Systems, (Vancuver, Canada), pp. 119 - 124, IEEE, 2001.
[38] Y. Kojima and Y. Yasumuro, "Hand Manipulation of Virtual Objects in Wearable Augmented Reality," in Virtual Systems and Multimedia, pp. 463 $-469,2001$.

[39] K. Aizawa, K. Ishijima, and M. Shiina, "Summarizing Wearable Video," in International Conference on Image Processing, vol. 2, pp. 398-401, Ieee, 2001.

[40] H. W. Ng, Y. Sawahata, and K. Aizawa, "Summarization of Wearable Videos Using Support Vector Machine," in IEEE International Conference on Multimedia and Expo, vol. Aug, pp. 325-328, IEEE, 2002.

[41] J. Gemmell, R. Lueder, and G. Bell, "The Mylifebits Lifetime Store," in Transactions on Multimedia Computing, Communications and Applications, (New York, New York, USA), pp. 0-5, ACM Press, 2002.

[42] R. DeVaul, A. Pentland, and V. Corey, "The Memory Glasses: Subliminal Vs. Overt Memory Support with Imperfect Information," in IEEE International Symposium on Wearable Computers, pp. 146-153, Ieee, 2003.

[43] Y. Sawahata and K. Aizawa, "Wearable Imaging System for Summarizing Personal Experiences," Multimedia and Expo, vol. Jul, no. 1, pp. 1-45, 2003.

[44] T. Hori and K. Aizawa, "Context-based Video Retrieval System for the Life-log Applications," in International Workshop on Multimedia Information Retrieval, (New York, New York, USA), p. 31, ACM Press, 2003.

[45] R. Bane and T. Hollerer, "Interactive Tools for Virtual X-Ray Vision in Mobile Augmented Reality," in International Symposium on Mixed and Augmented Reality, pp. 231-239, Ieee, 2004.

[46] M. Kolsch and M. Turk, "Fast 2d Hand Tracking with Flocks of Features and Multi-cue Integration," in Computer Vision and Pattern Recognition Workshop, pp. 158-158, IEEE Comput. Soc, 2004.

[47] J. Gemmell, L. Williams, and K. Wood, "Passive Capture and Ensuing Issues for a Personal Lifetime Store," in Workshop on Continuous Archival and Retrieval of Personal Experiences, (New York, NY), pp. 48-55, 2004.

[48] S. Mann, "Continuous Lifelong Capture of Personal Experience with Eyetap," in Continuous Archival and Retrieval of Personal Experiences, (New York, New York, USA), pp. 1-21, ACM Press, 2004.

[49] W. Mayol and D. Murray, "Wearable Hand Activity Recognition for Event Summarization," in International Symposium on Wearable Computers, pp. 1-8, IEEE, 2005.

[50] L. Sun, U. Klank, and M. Beetz, "Eyewatchme3d Hand and Object Tracking for Inside out Activity Analysis," in Computer Vision and Pattern Recognition, pp. 9-16, 2009.

[51] R. Tenmoku, M. Kanbara, and N. Yokoya, "Annotating User-viewed Objects for Wearable Ar Systems," in International Symposium on Mixed and Augmented Reality, pp. 192-193, Ieee, 2005.

[52] D. Tancharoen, T. Yamasaki, and K. Aizawa, "Practical experience recording and indexing of Life Log video," in Workshop on Continuous Archival and Retrieval of Personal Experiences, (New York, New York, USA), p. 61, ACM Press, 2005

[53] K. Aizawa, "Digitizing Personal Experiences: Capture and Retrieval of Life Log," in International Multimedia Modelling Conference, pp. 10-15, Ieee, 2005.

[54] R. Bane and M. Turk, "Multimodal Interaction with a Wearable Augmented Reality System," Computer Graphics and Applications, vol. 26, no. 3, pp. 6271,2006

[55] M. Kölsch, R. Bane, T. Höllerer, and M. Turk, "Touching the Visualized Invisible: Wearable Ar with a Multimodal Interface," IEEE Computer Graphics and Applications, vol. Jun, no. 1, pp. 62-71, 2006

[56] R. Castle, D. Gawley, G. Klein, and D. Murray, "Video-rate Recognition and Localization for Wearable Cameras," in British Machine Vision Conference, (Warwick), pp. 112.1-112.10, British Machine Vision Association, 2007.

[57] R. Castle, D. Gawley, G. Klein, and D. Murray, "Towards Simultaneous Recognition, Localization and Mapping for Hand-held and Wearable Cameras," in Conference on Robotics and Automation, pp. 4102-4107, Ieee, Apr. 2007.

[58] J. Wu and A. Osuntogun, "A Scalable Approach to Activity Recognition Based on Object Use," in Internantional Conference on Computer Vision, (Rio de Janeiro), pp. 1-8, IEEE, 2007.

[59] A. Davison, I. Reid, N. Molton, and O. Stasse, "MonoSLAM: Real-time Single Camera SLAM.," IEEE Transactions on Pattern Analysis and Machine Intelligence, vol. 29, pp. 1052-67, June 2007.

[60] R. Castle, G. Klein, and D. W. Murray, "Video-rate Localization in Multiple Maps for Wearable Augmented Reality," in International Symposium on Wearable Computers, (Pittsburgh, PA), pp. 15-22, IEEE, 2008. 
[61] W. Yi and D. Ballard, "Recognizing Behavior in Hand-eye Coordination Patterns," International Journal of Humanoid Robotics, vol. 6, no. 3, pp. 337359, 2009.

[62] E. Spriggs, F. De La Torre, and M. Hebert, "Temporal Segmentation and Activity Classification from First-person Sensing," in Computer Vision and Pattern Recognition Workshops, pp. 17-24, IEEE, June 2009.

[63] S. Sundaram and W. Cuevas, "High Level Activity Recognition Using Low Resolution Wearable Vision," Computer Vision and Pattern Recognition Workshops, pp. 25-32, June 2009

[64] K. Makita, M. Kanbara, and N. Yokoya, "View Management of Annotations for Wearable Augmented Reality," in Multimedia and Expo, pp. 982-985, Ieee, June 2009.

[65] X. Ren and C. Gu, "Figure-ground Segmentation Improves Handled Object Recognition in Egocentric Video," in Conference on Computer Vision and Pattern Recognition, pp. 3137-3144, IEEE, June 2010.

[66] V. Dovgalecs, R. Megret, H. Wannous, and Y. Berthoumieu, "Semisupervised Learning for Location Recognition from Wearable Video," in International Workshop on Content Based Multimedia Indexing, pp. 1-6, Ieee, June 2010 .

[67] D. Byrne, A. Doherty, and C. Snoek, "Everyday Concept Detection in Visual Lifelogs: Validation, Relationships and Trends," Multimedia Tools and Applications, vol. 49, no. 1, pp. 119-144, 2010.

[68] M. Hebert and T. Kanade, "Discovering Object Instances from Scenes of Daily Living," in International Conference on Computer Vision, pp. 762-769, Ieee, Nov. 2011.

[69] A. Fathi, X. Ren, and J. Rehg, “Learning to Recognize Objects in Egocentric Activities," in Computer Vision and Pattern Recognition, (Providence, RI), pp. 3281-3288, IEEE, June 2011

[70] A. Doherty and N. Caprani, "Passively Recognising Human Activities Through Lifelogging," Computers in Human Behavior, vol. 27, no. 5, pp. 1948-1958, 2011.

[71] A. Fathi, A. Farhadi, and J. Rehg, "Understanding Egocentric Activities," in International Conference on Computer Vision, pp. 407-414, IEEE, Nov. 2011.

[72] O. Aghazadeh, J. Sullivan, and S. Carlsson, "Novelty Detection from an Ego-centric Perspective," in Computer Vision and Pattern Recognition, (Pittsburgh, PA), pp. 3297-3304, Ieee, June 2011.

[73] K. Kitani and T. Okabe, "Fast Unsupervised Ego-action Learning for First-person Sports Videos," in Computer Vision and Pattern Recognition, (Providence, RI), pp. 3241-3248, IEEE, June 2011.

[74] K. Yamada, Y. Sugano, and T. Okabe, "Can Saliency Map Models Predict Human Egocentric Visual Attention?," in Internantional Conference on Computer Vision, pp. 1-10, 2011.

[75] M. Devyver, A. Tsukada, and T. Kanade, "A Wearable Device for First Person Vision," in International Symposium on Quality of Life Technology, vol. Jul, pp. 1-6, 2011.

[76] A. Borji, D. Sihite, and L. Itti, "Probabilistic Learning of Task-Specific Visual Attention," in Computer Vision and Pattern Recognition, (Providence, RI), pp. 470-477, Ieee, June 2012.

[77] H. Pirsiavash and D. Ramanan, "Detecting Activities of Daily Living in First-Person Camera Views," in Computer Vision and Pattern Recognition, pp. 2847-2854, IEEE, June 2012

[78] A. Fathi, Y. Li, and J. Rehg, "Learning to Recognize Daily Actions Using Gaze," in European Conference on Computer Vision, (Florence, Itaty), pp. 314327, Georgia Institute of Technology, 2012.

[79] K. Kitani, "Ego-Action Analysis for First-Person Sports Videos," Pervasive Computing, vol. 11, no. 2, pp. 92-95, 2012.

[80] K. Ogaki, K. Kitani, Y. Sugano, and Y. Sato, "Coupling Eye-motion and Ego-motion Features for First-person Activity Recognition," in IEEE Computer Society Conference on Computer Vision and Pattern Recognition, pp. 1-7, Ieee, June 2012.

[81] R. Grasset, T. Langlotz, and D. Kalkofen, “Image-driven View Management for Augmented Reality Browsers," in ISMAR, pp. 177-186, Ieee, Nov. 2012.

[82] H. Park, E. Jain, and Y. Sheikh, “3d Social Saliency from Head-mounted Cameras," Advances in Neural Information Processing Systems, pp. 431-439, 2012.

[83] K. Yamada, Y. Sugano, T. Okabe, Y. Sato, A. Sugimoto, and K. Hiraki, "Attention Prediction in Egocentric Video Using Motion and Visual Saliency," in Pacific Rim Conference on Advances in Image and Video Technology, pp. 277288, 2012.
[84] H. Boujut, J. Benois-Pineau, and R. Megret, "Fusion of Multiple Visual Cues for Visual Saliency Extraction from Wearable Camera Settings with Strong Motion," Internantional Conference on Computer Vision, pp. 436-445, 2012.

[85] C. Li and K. Kitani, "Model Recommendation with Virtual Probes for Egocentric Hand Detection," in ICCV 2013, (Sydney), IEEE Computer Society, 2013.

[86] C. Li and K. Kitani, "Pixel-Level Hand Detection in Ego-centric Videos," in Computer Vision and Pattern Recognition, pp. 3570-3577, Ieee, June 2013

[87] H. Wang and X. Bao, "Insight: Recognizing Humans Without Face Recognition," in Workshop on Mobile Computing Systems and Applications, (New York, NY, USA), pp. 2-7, 2013.

[88] J. Zariffa and M. Popovic, "Hand Contour Detection in Wearable Camera Video Using an Adaptive Histogram Region of Interest," Journal of NeuroEngineering and Rehabilitation, vol. 10, no. 114, pp. 1-10, 2013.

[89] Y. Li, A. Fathi, and J. Rehg, "Learning to Predict Gaze in Egocentric Video," in International Conference on Computer Vision, pp. 1-8, Ieee, 2013.

[90] I. González Díaz, V. Buso, J. Benois-Pineau, G. Bourmaud, and R. Megret, "Modeling Instrumental Activities of Daily Living in Egocentric Vision as Sequences of Active Objects and Context for Alzheimer Disease Research," in International workshop on Multimedia indexing and information retrieval for healthcare, (New York, New York, USA), pp. 11-14, ACM Press, 2013.

[91] A. Fathi and J. Rehg, "Modeling Actions through State Changes," in Computer Vision and Pattern Recognition, pp. 2579-2586, Ieee, June 2013.

[92] J. Zhang, L. Zhuang, Y. Wang, Y. Zhou, Y. Meng, and G. Hua, "An Egocentric Vision Based Assistive co-robot.," Conference on Rehabilitation Robotics, vol. 2013, pp. 1-7, June 2013.

[93] M. Ryoo and L. Matthies, "First-Person Activity Recognition: What Are They Doing to Me?," in Conference on Computer Vision and Pattern Recognition, (Portland, OR, US), pp. 2730-2737, IEEE Comput. Soc, 2013.

94] F. Martinez, A. Carbone, and E. Pissaloux, "Combining First-person and Third-person Gaze for Attention Recognition," IEEE International Conference and Workshops on Automatic Face and Gesture Recognition (FG), pp. 1-6, Apr. 2013.

[95] T. Starner, "Project Glass: An Extension of the Self," Pervasive Computing vol. 12, no. 2, p. 125, 2013.

[96] S. Narayan, M. Kankanhalli, and K. Ramakrishnan, "Action and Interaction Recognition in First-Person Videos," in Computer Vision and Pattern Recognition, pp. 526-532, Ieee, June 2014.

[97] K. Matsuo, K. Yamada, S. Ueno, and S. Naito, "An Attention-Based Activity Recognition for Egocentric Video," in Computer Vision and Pattern Recognition, pp. 565-570, Ieee, June 2014.

[98] D. Damen and O. Haines, "Multi-User Egocentric Online System for Unsupervised Assistance on Object Usage," in European Conference on Computer Vision, 2014.

[99] Y. Poleg, C. Arora, and S. Peleg, "Temporal Segmentation of Egocentric Videos," in Computer Vision and Pattern Recognition, pp. 2537-2544, Ieee, June 2014.

[100] K. Zheng, Y. Lin, Y. Zhou, D. Salvi, X. Fan, D. Guo, Z. Meng, and S. Wang "Video-based Action Detection using Multiple Wearable Cameras," in Workshop on ChaLearn Looking at People, 2014.

[101] K. Zhan, S. Faux, and F. Ramos, "Multi-scale Conditional Random Fields for first-person activity recognition," in International Conference on Pervasive Computing and Communications, pp. 51-59, Ieee, Mar. 2014

[102] S. Alletto, G. Serra, S. Calderara, F. Solera, and R. Cucchiara, "From Ego to Nos-Vision: Detecting Social Relationships in First-Person Views," in Computer Vision and Pattern Recognition, pp. 594-599, Ieee, June 2014.

[103] S. Alletto, G. Serra, S. Calderara, and R. Cucchiara, "Head Pose Estimation in First-Person Camera Views," in International Conference on Pattern Recognition, p. 4188, IEEE Computer Society, 2014.

[104] S. Lee, S. Bambach, D. Crandall, J. Franchak, and C. Yu, "This Hand Is My Hand: A Probabilistic Approach to Hand Disambiguation in Egocentric Video," in Computer Vision and Pattern Recognition, (Columbus, Ohio), pp. 1-8, IEEE Computer Society, 2014.

[105] S. Han, R. Nandakumar, M. Philipose, A. Krishnamurthy, and D. Wetherall, "GlimpseData: Towards Continuous Vision-based Personal Analytics," in Workshop on physical analytics, vol. 40, (New York, New York, USA), pp. 3136, ACM Press, 2014.

[106] A. Scheck, "Seeing the (Google) Glass as Half Full," EMN, pp. 20-21, 2014. 
[107] J. Wang and C. Yu, "Finger-fist Detection in First-person View Based on Monocular Vision Using Haar-like Features," in Chinese Control Conference, pp. 4920-4923, Ieee, July 2014.

[108] Y. Liu, Y. Jang, W. Woo, and T.-K. Kim, "Video-Based Object Recognition Using Novel Set-of-Sets Representations," in Computer Vision and Pattern Recognition, pp. 533-540, Ieee, June 2014.

[109] S. Feng, R. Caire, B. Cortazar, M. Turan, A. Wong, and A. Ozcan, "Immunochromatographic Diagnostic Test Analysis Using Google Glass.," ACS nano, vol. 1, Feb. 2014.

[110] Y. Poleg, C. Arora, and S. Peleg, "Head Motion Signatures from Egocentric Videos," in Asian Conference on Computer Vision, vol. Nov, (Singapore), pp. 1-15, Springer, 2014

[111] G. Rogez and J. S. III, “3D Hand Pose Detection in Egocentric RGB-D Images," in ECCV Workshop on Consumer Depth Camera for Computer Vision, vol. Sep, (Zurich, Switzerland), pp. 1-14, Springer, Nov. 2014.

[112] G. Rogez, J. S. Supancic, and D. Ramanan, “Egocentric Pose Recognition in Four Lines of Code," in Computer Vision and Pattern Recognition, vol. Jun, pp. 1-9, Nov. 2015.

[113] R. Templeman, M. Korayem, D. Crandall, and K. Apu, "PlaceAvoider: Steering first-person cameras away from sensitive spaces," in Network and Distributed System Security Symposium, no. February, pp. 23-26, 2014.

[114] V. Buso, J. Benois-Pineau, and J.-P. Domenger, “Geometrical Cues in Visua Saliency Models for Active Object Recognition in Egocentric Videos," in International Workshop on Perception Inspired Video Processing, (New York, New York, USA), pp. 9-14, ACM Press, 2014.

[115] B. Xiong and K. Grauman, "Detecting Snap Points in Egocentric Video with a Web Photo Prior," in Internantional Conference on Computer Vision, 2014.

[116] W. Min, X. Li, C. Tan, B. Mandal, L. Li, and J. H. Lim, “Efficient Retrieval from Large-Scale Egocentric Visual Data Using a Sparse Graph Representation," in Computer Vision and Pattern Recognition Workshops, pp. 541-548, Ieee, June 2014

[117] M. Bolanos, M. Garolera, and P. Radeva, "Video Segmentation of LifeLogging Videos," in Articulated Motion and Deformable Objects, (Palma de Mallorca, Spain), pp. 1-9, Springer Verlag, 2014.

[118] J. W. Barker and J. W. Davis, "Temporally-Dependent Dirichlet Process Mixtures for Egocentric Video Segmentation," in Computer Vision and Pattern Recognition, pp. 571-578, Ieee, June 2014.

[119] M. Okamoto and K. Yanai, "Summarization of Egocentric Moving Videos for Generating Walking Route Guidance," in Image and Video Technology, pp. 431-442, 2014.

[120] I. Arev, H. S. Park, Y. Sheikh, J. Hodgins, and A. Shamir, "Automatic Editing of Footage from Multiple Social Cameras," ACM Transactions on Graphics, vol. 33, pp. 1-11, July 2014.

[121] M. Jones and J. Rehg, "Statistical Color Models with Application to Skin Detection 2 Histogram Color Models," in Computer Vision and Pattern Recognition, vol. Jun, (Fort Collins, CO), pp. 1-23, IEEE Computer Society, 1999.

[122] M. Schlattmann, F. Kahlesz, R. Sarlette, and R. Klein, "Markerless 4 Gestures 6 Dof Real-time Visual Tracking of the Human Hand with Automatic Initialization," Computer Graphics Forum, vol. 26, pp. 467-476, Sept. 2007.

[123] M. Schlattman and R. Klein, "Simultaneous 4 Gestures 6 Dof Real-time Two-hand Tracking Without Any Markers," in Symposium on Virtual Reality Software and Technology, (New York, NY, USA), pp. 39-42, ACM Press, 2007.

[124] J. Rehg and T. Kanade, “DigitEyes: Vision-Based Hand Tracking for Human-Computer Interaction," in Workshop on Motion of Non-Rigid and Articulated Bodies, pp. 16-22, IEEE Comput. Soc, 1994.

[125] P. Morerio, G. C. Georgiu, L. Marcenaro, and C. Regazzoni, “Optimizing superpixel clustering for real-time egocentric-vision applications," IEEE Signal Processing Letters, 2014.

[126] V. Bettadapura, I. Essa, and C. Pantofaru, "Egocentric Field-of-View Localization Using First-Person Point-of-View Devices," in Winter Conference on Applications of Computer Vision, vol. Jan, (Waikoloa, HI), pp. 626-633, 2015.

[127] Y. Hoshen and S. Peleg, "Egocentric Video Biometrics," arXiv preprint, Nov. 2015.

[128] M. Spain and P. Perona, "Measuring and Predicting Object Importance," International Journal of Computer Vision, vol. 91, pp. 59-76, Aug. 2010.

[129] T. Berg and D. Forsyth, "Animals on the Web," in Computer Vision and Pattern Recognition, vol. 2, pp. 1463-1470, IEEE, 2006.
[130] C. Yu and D. Ballard, "Learning To Recognize Human Action Sequences," in Development and Learning, pp. 28-33, 2002.

[131] N. Krahnstoever, J. Rittscher, P. Tu, K. Chean, and T. Tomlinson, “Activity Recognition using Visual Tracking and RFID," in IEEE Workshops on Applications of Computer Vision (WACV/MOTION'05), vol. 1, (Breckenridge, CO), pp. 494-500, IEEE, Jan. 2005.

[132] D. Patterson, D. Fox, H. Kautz, and M. Philipose, "Fine-Grained Activity Recognition by Aggregating Abstract Object Usage," in Ninth IEEE International Symposium on Wearable Computers (ISWC'05), pp. 44-51, IEEE, 2005.

[133] M. Philipose and K. Fishkin, "Inferring Activities from Interactions with Objects," Pervasive Computing, vol. 3, no. 4, pp. 50-57, 2004.

[134] M. Kolsch, M. Turk, and T. Hollerer, "Vision-based Interfaces for Mobility," in Mobile and Ubiquitous Systems: Networking and Services, pp. 86 - 94, Ieee, 2004.

[135] G. Riva, F. Vatalaro, F. Davide, and M. Alcaiz, eds., Ambient Intelligence The Evolution of Technology, Communication and Cognition Towards the Future of Human-Computer Interaction, vol. 6. IEEE, January 2005.

[136] J. Garca-Rodrguez and J. M. García-Chamizo, "Surveillance and humancomputer interaction applications of self-growing models," Applied Soft Computing, vol. 11, no. 7, pp. 4413 - 4431, 2011. Soft Computing for Information System Security.

[137] M. Morshidi and T. Tjahjadi, “Gravity Optimised Particle Filter for Hand Tracking," Pattern Recognition, vol. 47, no. 1, pp. 194-207, 2014.

[138] V. Spruyt, A. Ledda, and W. Philips, "Real-time, Long-term Hand Tracking with Unsupervised Initialization," in International Conference on Image Processing, (Melbourne, Australia), IEEE Comput. Soc, 2013.

[139] C. Shan, T. Tan, and Y. Wei, "Real-time Hand Tracking Using a Mean Shift Embedded Particle Filter," Pattern Recognition, vol. 40, pp. 1958-1970, July 2007.

[140] G. Ben-Artzi, M. Werman, and S. Peleg, "Event Matching from Significantly Different Views using Motion Barcodes," arXiv preprint, Dec. 2015.

[141] D. Lu and Q. Weng, “A Survey of Image Classification Methods and Techniques for Improving Classification Performance," International Journal of Remote Sensing, vol. 28, pp. 823-870, Mar. 2007.

[142] S. Chiappino, L. Marcenaro, P. Morerio, and C. Regazzoni, “Event Based Switched Dynamic Bayesian Networks for Autonomous Cognitive Crowd Monitoring," in Augmented Vision and Reality, Augmented Vision and Reality, pp. 1-30, Springer Berlin Heidelberg, 2013.

[143] F. Camastra and A. Vinciarelli, Machine Learning for Audio, Image and Video Analysis: Theory and Applications. Springer, 2007.

[144] R. Achanta, A. Shaji, K. Smith, A. Lucchi, P. Fua, and S. Susstrunk "Slic superpixels compared to state-of-the-art superpixel methods," Pattern Analysis and Machine Intelligence, IEEE Transactions on, vol. 34, no. 11, pp. 2274-2282, 2012.

[145] P. Morerio, L. Marcenaro, and C. S. Regazzoni, "A generative superpixel method," in 17th IEEE International Conference on Information Fusion (FUSION 2014), 2014

[146] Y. J. Lee and K. Grauman, "Predicting Important Objects for Egocentric Video Summarization," International Journal of Computer Vision, vol. Jan, pp. 1-19, Jan. 2015.

[147] A. Knittel, "Learning Feature Hierarchies under Reinforcement," in Evolutionary Computation (CEC), 2012 IEEE Congress on, pp. 1-8, June 2012.

[148] C. Tan, H. Goh, and V. Chandrasekhar, "Understanding the Nature of First-Person Videos: Characterization and Classification using Low-Level Features," in Computer Vision and Pattern Recognition, pp. 535-542, 2014.

[149] S. Chiappino, P. Morerio, L. Marcenaro, and C. S. Regazzoni, “A bioinspired Knowledge Representation Method for Anomaly Detection in Cognitive Video Surveillance Systems," in Information Fusion (FUSION), 2013 16th International Conference on, pp. 242-249, July 2013.

[150] S. Chiappino, P. Morerio, L. Marcenaro, and C. Regazzoni, “Bio-inspired Relevant Interaction Modelling in Cognitive Crowd Management," Journal of Ambient Intelligence and Humanized Computing, pp. 1-22, 2014. 


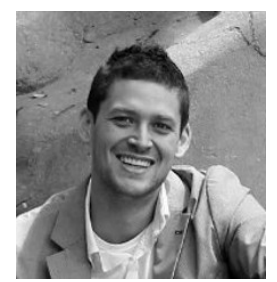

Alejandro Betancourt Alejandro Betancourt is $\mathrm{PhD}$ candidate of the Interactive and Cognitive Environment program between the Universita degli Studi di Genova and the Eindhoven University of Technology. Alejandro is a Mathematical Engineer and Master In Applied Mathematics from EAFIT University (Medellin, Colombia). Since 2011 Alejandro has been involved in research about Artificial Intelligence, Machine Learning and Cognitive Systems.

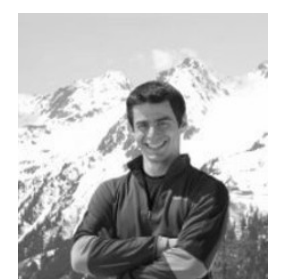

Pietro Morerio Pietro Morerio received his B.SC in Physics from the Faculty of Science, University of Milan (Italy) in 2007. In 2010 he received from the same university his M. Sc. in Theoretical Physics (summa cum laude). He was Research Fellow at the University of Genoa (Italy) from 2011 to 2012, working in Video Analysis for Interactive Cognitive Environments. Currently, he is pursuing a PhD degree in Computational Intelligence at the same institution.

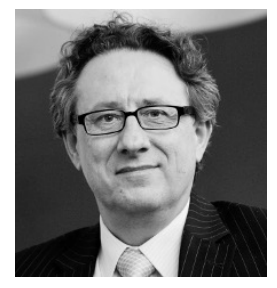

Matthias Rauterberg Matthias Rauterberg is professor at the department of Industrial Design and the head of the Designed Intelligence group at Eindhoven University of Technology (The Netherlands). Matthias received the B.S. in Psychology (1978) at the University of Marburg (Germany), the B.S. in Philosophy (1981) and Computer Science (1983), the M.S. in Psychology (1981, summa cum laude) and Computer Science (1986, summa cum laude) at the University of Hamburg (Germany), and the Ph.D. in Computer Science/Mathematics (1995, awarded) at the University of Zurich (Switzerland).

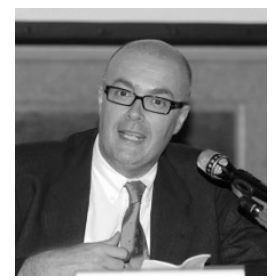

Carlo Regazzoni Carlo S. Regazzoni received the Laurea degree in Electronic Engineering and the Ph.D. in Telecommunications and Signal Processing from the University of Genoa (UniGE), in 1987 and 1992, respectively. Since 2005 Carlo is Full Professor of Telecommunications Systems. Dr. Regazzoni is involved in research on Signal and Video processing and Data Fusion in Cognitive Telecommunication Systems since 1988. 